\title{
Fighting COVID-19 at Purdue University: Design and Evaluation of a Game for Teaching COVID-19 Hygienic Best Practices
}

\author{
Dominic Kao \\ Purdue University \\ West Lafayette, Indiana, USA \\ kaod@purdue.edu \\ Abhigna Peddireddy \\ Purdue University \\ West Lafayette, Indiana, USA \\ apeddir@purdue.edu \\ John Springer \\ Purdue University \\ West Lafayette, Indiana, USA \\ jaspring@purdue.edu
}

\author{
Amogh Joshi \\ Purdue University \\ West Lafayette, Indiana, USA \\ joshi134@purdue.edu \\ Arjun Kramadhati Gopi \\ Purdue University \\ West Lafayette, Indiana, USA \\ akramadh@purdue.edu \\ Bethany S. McGowan \\ Purdue University \\ West Lafayette, Indiana, USA \\ bmcgowa@purdue.edu
}

\author{
Christos Mousas \\ Purdue University \\ West Lafayette, Indiana, USA \\ cmousas@purdue.edu \\ Jianyao Li \\ Purdue University \\ West Lafayette, Indiana, USA \\ li3682@purdue.edu \\ Jason B. Reed \\ Purdue University \\ West Lafayette, Indiana, USA \\ reed252@purdue.edu
}

\begin{abstract}
COVID-19 has upended lives everywhere, causing millions of deaths and tens of millions of infections worldwide. Nevertheless, for many people, staying in permanent isolation is neither desirable nor possible. To mitigate the spread of the disease, we iteratively developed a game that teaches hygienic best practices for preventing COVID-19. We consulted professional game designers, health experts, and educational technology designers. We then compared the effectiveness of the game to an equivalent video in two longitudinal experiments during the pandemic: 1) an experiment in a programming lab ( $\mathrm{N}=11)$, and 2) an online-only experiment $(\mathrm{N}=475)$. In Experiment \#1, we observe that participants in the game condition had higher intrinsic motivation, and a more sustained rise in hygienic self-efficacy, compared to participants in the video condition. Both conditions saw a rise in COVID-19 knowledge and positive hygienic attitude. Both conditions were relatively unchanged in COVID-19 anxiety and hygienic behavior. In Experiment \#2, participants in the game condition experienced greater intrinsic motivation than participants in the video condition. Both conditions saw a sustained rise in COVID-19 hygienic self-efficacy, positive hygienic attitudes, and knowledge. Neither condition saw an effect on COVID-19 anxiety. Our work demonstrates that game-based learning can be an effective approach for teaching COVID-19 hygienic knowledge, for improving COVID-19 hygienic self-efficacy, and for fostering COVID-19 hygienic positive attitudes, and is more intrinsically motivating than video-based learning.
\end{abstract}

This work is licensed under a Creative Commons Attribution-Share Alike International 4.0 License.

FDG'21, August 3-6, 2021, Montreal, QC, Canada

(c) 2021 Copyright held by the owner/author(s).

ACM ISBN 978-1-4503-8422-3/21/08.

https://doi.org/10.1145/3472538.3472552

\section{CCS CONCEPTS}

- Human-centered computing $\rightarrow$ Human computer interaction (HCI).

\section{KEYWORDS}

Games for health; COVID-19; coronavirus

\section{ACM Reference Format:}

Dominic Kao, Amogh Joshi, Christos Mousas, Abhigna Peddireddy, Arjun Kramadhati Gopi, Jianyao Li, John Springer, Bethany S. McGowan, and Jason B. Reed. 2021. Fighting COVID-19 at Purdue University: Design and Evaluation of a Game for Teaching COVID-19 Hygienic Best Practices. In The 16th International Conference on the Foundations of Digital Games (FDG) 2021 (FDG'21), August 3-6, 2021, Montreal, QC, Canada. ACM, New York, NY, USA, 23 pages. https://doi.org/10.1145/3472538.3472552

\section{INTRODUCTION}

As of January 2021, there have been upwards of 91 million cases of COVID-19 and over 1.95 million deaths [66]. Nearly 1,000 universities re-opened in August and September of 2020 [45], which challenged both educators and students alike to create safe, highquality, instructional environments. The stakes have never been higher, as COVID-19 can lead to short- and long-term cardiovascular, respiratory, renal, dermatologic, neurological, and psychiatric consequences [19]. Nevertheless, there exist gaps in both knowledge and protective behaviors [9]. For example, a March 2020 survey of 1,404 medical students in Jordan found that only $41.8 \%$ of respondents believed COVID-19 to be airborne, and only $9.7 \%$ believed wearing masks would help prevent the spread of COVID-19 [39]. While partially a reflection of the rapidly changing nature of coronavirus information (and at times, misinformation [11]), it is important now more than ever before to disseminate COVID-19 best practices.

A well-designed game may be able to effectively teach players COVID-19 hygienic best practices. Through fostering knowledge about COVID-19 and best practices in behavior and sanitization 
through gameplay, this can in principle reduce the chances of players becoming infected and of infecting others. There is, however, currently scarce evidence that a serious game can successfully teach COVID-19 hygienic best practices. A game called Escape COVID19 was previously created for health care workers to learn about about COVID-19 prevention and control, but the game is yet to be empirically tested [58]. Here, we describe the iterative design and development of a COVID-19 game called Fighting COVID-19 at Purdue University, wherein the goal is to teach players COVID-19 hygienic best practices. We assess the impact of playing the game in two longitudinal three-week studies. In study one, we test the game in a programming class taking place in-person during the pandemic. In study two, we test the game on Amazon Mechanical Turk (AMT) for a larger sample size. We had 5 research questions:

RQ1: How does a COVID-19 game compare against a video in generating intrinsic motivation?

RQ2: How does a COVID-19 game compare against a video in affecting COVID-19 anxiety?

RQ3: How does a COVID-19 game compare against a video in affecting hygienic self-efficacy?

RQ4: How does a COVID-19 game compare against a video in affecting positive hygienic attitude?

RQ5: How does a COVID-19 game compare against a video in teaching COVID-19 knowledge?

In the in-person study, we observe that participants in the game condition had higher intrinsic motivation, as well as a more sustained rise in hygienic self-efficacy, than participants in the video condition. Both conditions saw a rise in COVID-19 knowledge and positive hygienic attitude. Both conditions were relatively unchanged in COVID-19 anxiety and hygienic behavior. In the AMT online study, participants in the game condition experienced greater intrinsic motivation than participants in the video condition. Both conditions saw a sustained rise in COVID-19 hygienic self-efficacy, positive hygienic attitudes, and knowledge. Neither condition saw an effect on COVID-19 anxiety. Our studies show that game-based learning can be an effective approach for teaching COVID-19 hygienic knowledge, for improving COVID-19 hygienic self-efficacy, and for fostering COVID-19 hygienic positive attitudes, and is more intrinsically motivating than video-based learning.

\section{RELATED WORK}

\subsection{Hygiene and Digital Interventions}

Poor hygiene contributes to various diseases such as diarrhea, influenza, and skin infections [14]. Interventions designed to develop hygienic best practices have shown to reduce infections $[8,67]$ and mortality [13]. Digital mediums have an advantage over traditional means when delivering health interventions in terms of efficiency, scale, and reaching remote populations. Several meta-reviews have found a small, positive impact of digital interventions [64], suggesting that improving health outcomes is possible through digital media.

Two previous studies [30,61] aimed to improve handwashing frequency in different targeted populations using instant messaging (e.g., WeChat). One study aimed to improve handwashing behavior in children before eating and after using restroom facilities [30]. A population afflicted with Hand, Foot, and Mouth Disease (HFMD) received instructional material and reminders to apply medication through WeChat. They showed a significant decrease in rashes and recovery period [30] compared to the group receiving a face-toface intervention. The other study focused on improving mothers' handwashing behavior before interacting with their newborn baby [61]. Both studies showed a significant increase in handwashing frequency for the experimental group compared to the control group. In a similar vein, an online intervention [44] was targeted at improving the frequency of handwashing, attitudes, and beliefs towards hygiene. The participants who completed the instructional materials available on a website reported decreased respiratory and gastrointestinal infections. Therefore, digital media appears effective at engendering hygienic outcomes.

\subsection{Games for Health}

Video games can keep players engaged and intrinsically motivated [27], which may offer an advantage in delivering interventions compared to traditional methods [38, 43]. When positive, desirable behaviors are rewarded in games, this may help players learn more effectively due to positive reinforcement [53]. To this effect, many educational video games have successfully taught essential concepts in the field of computer science [37, 48], biology [49], pharmacy [41], physical health [16], mental health [62], and areas like formal interview preparation [42]. Health interventions delivered through video games typically use a narrative plot [60] or non-player characters (NPCs) [56] to teach instructional content. In one study on adolescents and young adults with cancer, a video game increased adherence to medications, self-efficacy, and cancer knowledge [38]. Other games have focused on improving glucose monitoring [34] and cultivating behaviors like increasing physical activity and changing diet to increase water, fruits, and vegetable intake [12]. Although an increasing number of studies look at improving health outcomes through games, only a handful of studies have addressed personal hygiene through a video game intervention.

A majority of interventions focus on gamifying handwashing procedures outlined by the World Health Organization (WHO) [68]. The game Soap and Water [36] used motion sensors (Kinect V2) to gamify handwashing for children with intellectual disabilities. Participants perform handwashing, which is captured and rendered on the screen to give immediate feedback. The rendered projection also contains stubborn enemies (representing germs) present on the hands. To "kill" the enemies, players must correctly follow the handwashing procedure. The results indicate that the game helped participants improve correctness in following handwashing procedure steps. CatCare [35] was a game designed to simulate a veterinarian clinic where the player isolates cats with different diseases. The player wins the game if they take proper precautions while handling the sick cats-such as appropriate handwashing after touching sick cats-and lose the game if they transmit the infection to other healthy cats. The game also simulated stressful situations by creating time pressure for each of the activities. The game $e-B u g$ was developed to teach hygiene concepts to school children [29]. The game environment was the human body, and the player controlled a character that fired soap bubbles at microbes and navigated other hazards. The learning content was delivered through the game 
mechanics, such as (1) reducing the character's size to a microscopic level to understand invisible microbes' presence and (2) firing soap bubbles to "destroy" the enemies that represented harmful microbes. Middle-school children played the game online and experienced a significant improvement in knowledge regarding the concepts of germs, handwashing, and antibiotics. SureWash is an automated tool to increase handwashing compliance in hospitals by gamifying handwashing [33]. The tool gives a percentage score based upon the cleanliness of the hands after washing. In a similar vein, a virtual reality trainer prototype was created with a focus to highlight hand-to-hand transmission of diseases in hospitals [24]. Escape COVID-19 was developed for health care workers to learn more about preventing the transmission of COVID-19 [58]. Our goal in this paper is to develop a COVID-19 game that teaches hygienic best practices and to empirically study the game's effects.

\subsection{Environmental Sanitization}

Recent studies have emphasized the need to create hygiene interventions that consider environmental sanitization $[1,21,26]$ in conjunction with personal hygiene. Environmental sanitization is important to consider because viruses and harmful bacteria can live on surfaces for several hours to days [21]. For example, one possible transmission path for infection of communicable diseases is through hand contact with the mouth after touching a frequentlyused object or space. The Centers for Disease Control and Prevention (CDC) has released new guidelines outlining best practices to combat COVID-19, and these guidelines include disinfecting commonly-used objects and surfaces [21]. Companies and educational institutions remaining open during the pandemic also employ these practices to combat COVID-19 [2,3].

Examples of frequently-used spaces include public computers and shared workspaces, which are ubiquitous in most educational institutions. Several studies have focused on pathogen transmission and found a $30-60 \%$ prevalence rate of harmful bacteria and viruses on computer keyboards and mice in shared computer spaces in universities [6, 23]. Moreover, recent studies also highlight public computers and shared workspaces as a possible transmission space for COVID-19, specifically [31, 69]. A recent systematic review [57] indicated that only two studies employed a multi-faceted approach (i.e., including environment disinfection in addition to personal hygiene) to hygiene intervention. Both these studies $[54,63]$ saw improved health outcomes in participants and reduced prevalence of infectious viruses (e.g., Norovirus) on shared surfaces, such as desks, in the experimental group.

COVID-19 has forced society to recognize the risk of everyday objects and places, as well as to develop new behaviors to reduce risks, while using shared workspaces and public computers. However, procedures for how to best sanitize a shared space or the optimal hygienic behaviors to adopt is often assumed to be common knowledge or left to be learned by oneself. Therefore, we developed a game called Fighting COVID-19 at Purdue University, which is, to the best of our knowledge, the first game that adopts both personal and environmental hygienic procedures to attempt to reduce the risk of COVID-19 transmission.

\section{THE GAME: FIGHTING COVID-19 AT PURDUE UNIVERSITY}

Our primary goal was to develop a game to teach critical COVID-19 knowledge and best practices. To do so, we decided to set the game in a virtual university computer lab based on the real lab where we would run our first study. (See Figure 1.) The completed game can be played in its entirety (see footnote ${ }^{12}$ ). The game development team consisted of 2 lab teaching assistants who both had several months of experience administering programming labs during the pandemic, 2 game designers, 2 health science experts who were both faculty members, and 1 educational technology designer. All team members participated in the iterative design and development of the game.

The team first decided on the knowledge that we collectively felt was the most crucial knowledge to communicate to the player. This was first done individually, each team member brainstormed and developed a list of what they felt was most important to teach the player. The team then came together, and based on overlapping ideas, we reached a consensus on what the game should teach. We then developed a set of facts that we wanted players to be able to answer correctly after playing the game. These were based on the official Centers for Disease Control and Prevention (CDC) website and then validated by our health sciences experts. We then collectively developed an initial storyboard, which was a general outline of the gameplay. We began game development in late September 2020 , and we needed to complete the game by mid-October 2020 in order to deploy an in-class experiment during the semester. Given this short time frame, we adopted a highly compressed Agile software development process in which new game prototypes were released every few days, after which all team members provided extensive feedback. In this development time frame, we had 7 major iterations in which all team members provided input/feedback on increasingly complex versions of the game, with dozens of minor iterations in which individual subsets of the team discussed how best to proceed.

The game uses a vector art style and is developed in Construct 3, an HTML5-based 2D game editor, in order to maximize portability. The game is playable in any modern browser, on any type of desktop machine. A teacher-like character guides the player throughout the game with a voiceover. Gameplay is based on first-person dungeon crawler role-playing video games (RPGs), wherein the player clicks to move or interact with the game world. (See Figure 2.) The game contains five challenges, which are the main gameplay elements that teach the player about COVID-19: 1) mask wearing, 2) social distancing, 3) disinfecting a workstation, 4) COVID-19 symptoms and quarantining, and 5) handwashing. The challenges vary in their gameplay mechanics, but all of them involve using the mouse and clicking. One specific design decision we made was to prevent players from skipping the audio dialogue before the teacher had finished speaking. We did this to prevent players from skipping important instructional content embedded in the game.

\footnotetext{
${ }^{1}$ Video of Gameplay: https://youtu.be/v6lvV9ujxC4

${ }^{2}$ Playable Game: https://web.ics.purdue.edu/ kaod/COVIDGame/
} 


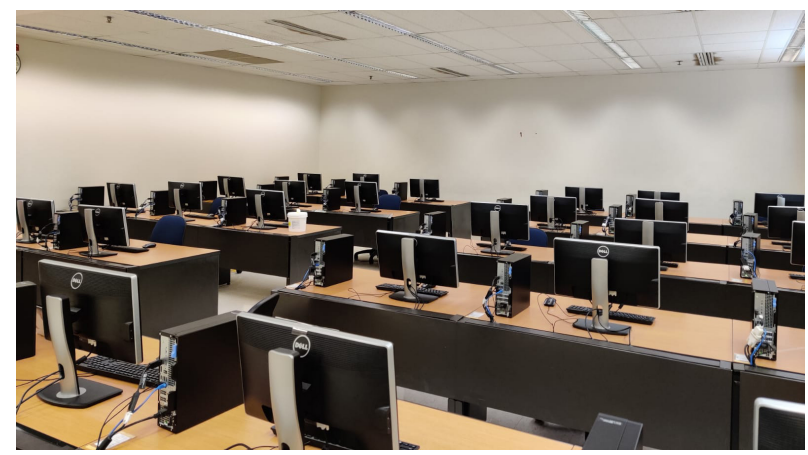

(a) Real-life lab classroom

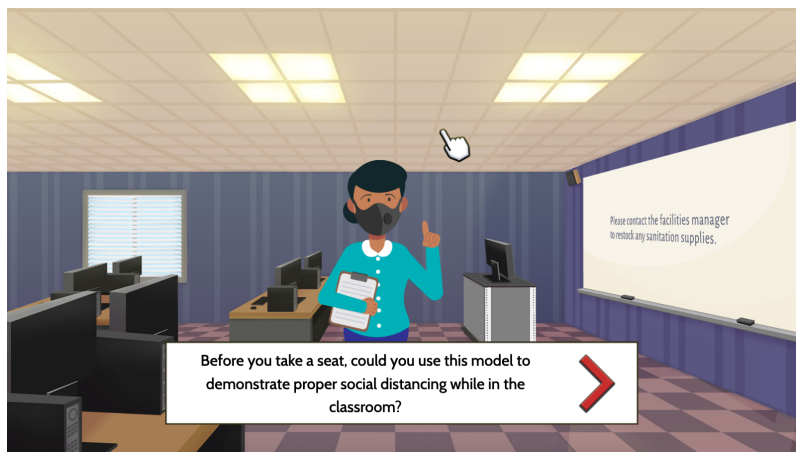

(b) In-game lab classroom

Figure 1: Real-life and in-game lab classrooms. Importantly, although the the game takes place in a university setting, the concepts taught in the game are generally relevant both in and outside of a university setting.

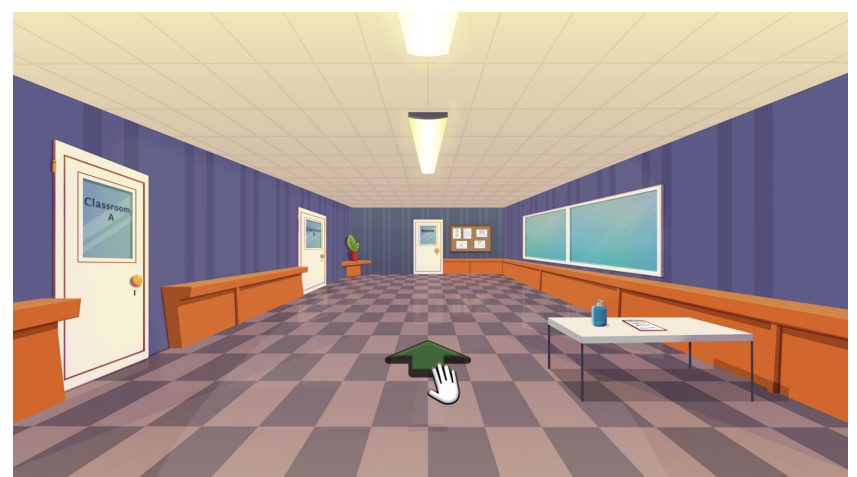

Figure 2: The game features RPG dungeon crawler-style mechanics with point-and-click for navigation and interaction.

\subsection{Mask wearing}

The first challenge starts with a prompt by the instructor to help other students correctly wear a mask. Participants use the mouse to click-and-drag to reposition masks over each student's face. There are specific hints given to the player as each of the faces depicts various incorrect positions to wear a mask (see Figure 3). When the participant correctly repositions the mask, a distinctive audio tone notifies the participant of their correct answer.

\subsection{Social Distancing}

The second challenge asks players to place students into appropriate socially-distanced positions. The participants drag-and-drop virtual students into lab classroom seats (see Figure 4). Placing a student too close to another results in both a pop-up message and a buzzer sound effect indicating the incorrect action.

\subsection{Disinfecting a Workstation}

The third challenge focuses on disinfecting a computer workstation. This scenario was created to mimic the workstations that students might see in a university computer lab, but also generalizes to workstations at the office or home. We target two instructional objectives here: 1) Frequently touched areas on a workstation may contain pathogens. Research shows that public workspaces are a major transmission space for COVID-19 [32, 70], with several studies finding a $30-60 \%$ prevalence rate of pathogens on computer keyboards and mice in shared computer spaces in universities [5, 22]. To demonstrate this to players, we made the virus visible on different surfaces. 2) The second objective was to promote an understanding of how to properly disinfect surfaces. We ask the player to use multiple disinfectant wipes to wipe down the workstation. Used disinfectant wipes must be disposed in the trash, or the dirty wipes spawn additional viruses where they were left. Instructional messages are displayed to the player during the cleaning process. See Figure 5.

\subsection{COVID-19 Symptoms and Quarantining}

The fourth challenge focuses on helping the player understand the common symptoms of COVID-19 and that it spreads through close proximity with other people. In an early iteration of the game, we showed a social simulation with people that moved and the player was required to construct quarantine barriers. However, we felt this was too attention demanding if we were simultaneously delivering instructional content. We instead developed a challenge with stationary faces representing people. Some of the faces exhibit symptoms of COVID-19, and players must quarantine the symptomatic individual and those who were in close proximity to that individual. The intensity of the symptom was denoted by the color of the face (yellow for mild, red for severe), with an image indicating the specific symptom being exhibited. See Figure 6.

\subsection{Handwashing}

The fifth and final challenge consists of players demonstrating proper handwashing. Participants need to first wet their hands under water, lather their hands with soap, scrub for 20 seconds, rinse their hands, and then dry their hands using a clean towel or air dryer. This is based on CDC's Five Steps to Wash Your Hands the Right Way [20]. Players control their hand movements using the mouse, while instructions printed on the wall provide step-by-step guidance. A timer ensured players scrubbed their hands (by holding down the left mouse button) for 20 seconds and only counted down while the player was scrubbing. The countdown timer would reset 

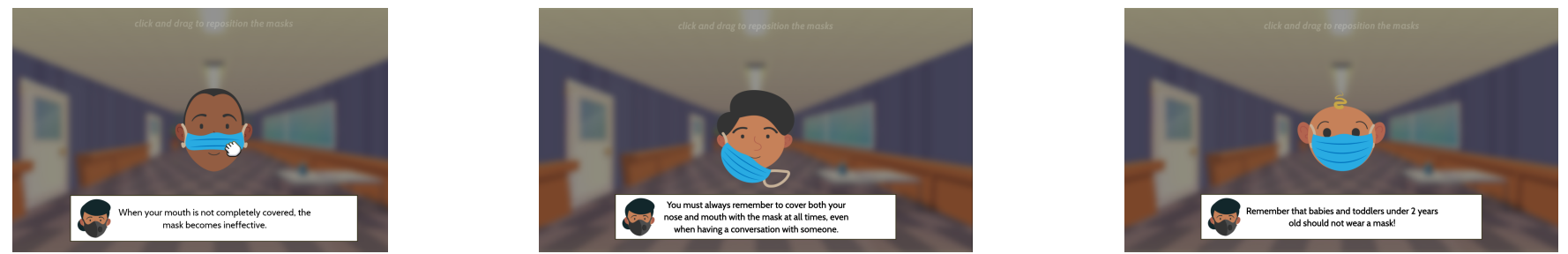

Figure 3: In the mask-wearing challenge, players use the mouse to click-and-drag incorrectly positioned masks into their correct positions.
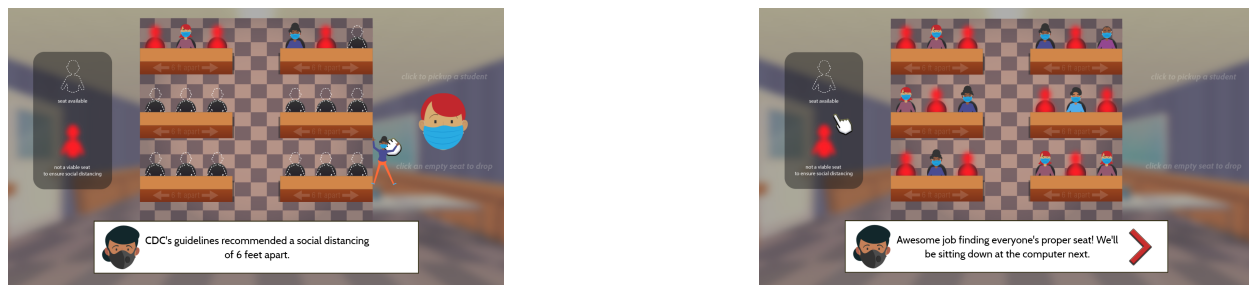

Figure 4: In the social distancing challenge, participants drag-and-drop students into an appropriately social-distanced seating arrangement.
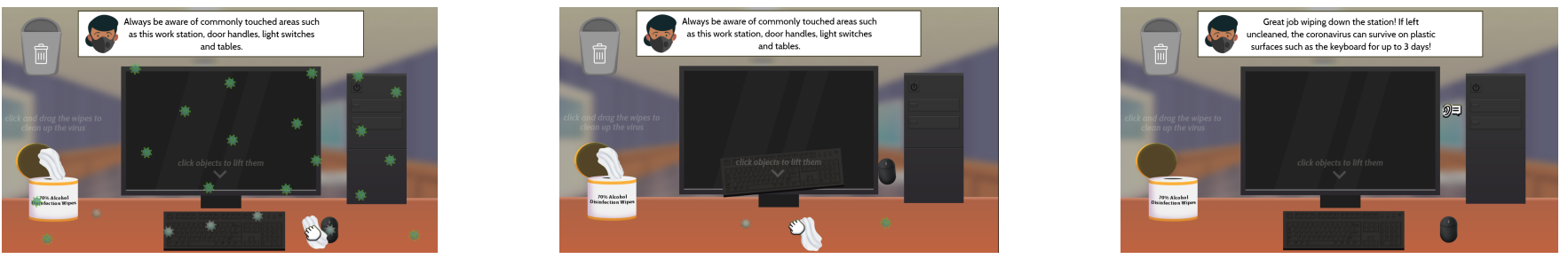

Figure 5: In the disinfecting a workstation challenge, players grab disinfectant wipes and drag them across different surfaces. Players must click on the keyboard and mouse to clean underneath them.
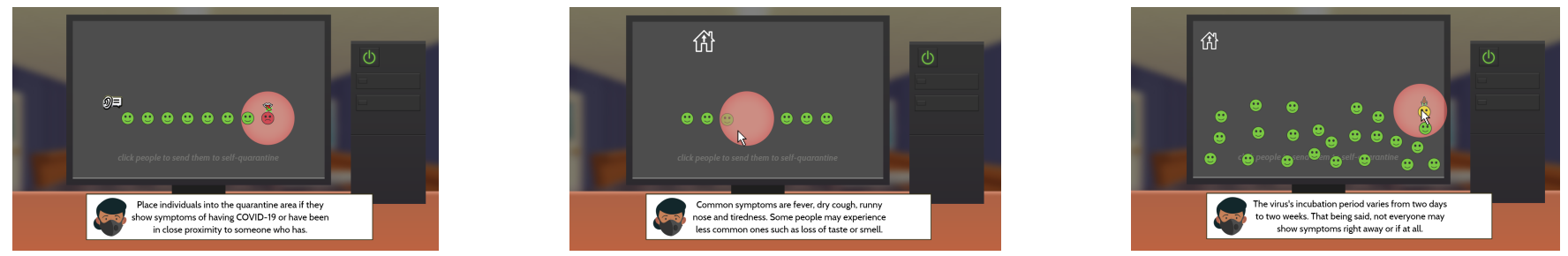

Figure 6: In the COVID-19 symptoms and quarantining challenge, players quarantine individuals showing symptoms of COVID-19, as well as those individuals who were in close proximity.

if the player's hands were rinsed before the timer was completed. Hand animations during scrubbing were meant to demonstrate that scrubbing involved thoroughly reaching all areas of both hands. See Figure 7.

\section{THE VIDEO}

Our goal in creating a video was to make it as identical as possible to the game in terms of instructional content and to ensure that it was of a similar quality level as the game. The completed video can be watched here (see footnote ${ }^{3}$ ). To create the video, we hired a professional instructional designer with over 20 years of experience in developing instructional content. The designer was compensated $\$ 100$ USD/hr. The instructional designer first became familiar with the game by documenting the content in the game. To maintain as much similarity as possible with the game, the team decided to use the existing graphics, animations, and audio from the game to develop the video. As with the game, the video was iterated on several times with feedback from the team. The health sciences

\footnotetext{
${ }^{3}$ Video Condition: https://youtu.be/VdeIZ0mMe1E
} 

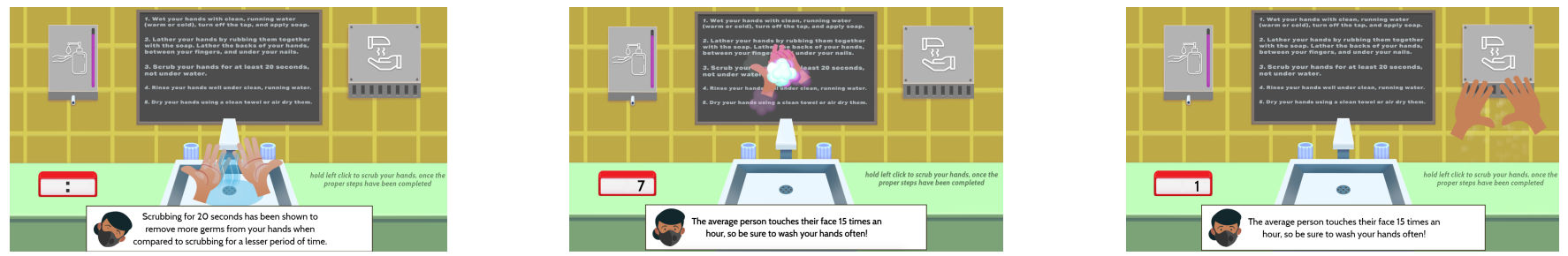

Figure 7: In the handwashing challenge, players must follow CDC's Five Steps to Wash Your Hands the Right Way [20].

experts on the team ensured that the video was accurate in content and contained the same content as the game. One important decision was whether to create a video that would last approximately as long as the game (which took around 10-15 minutes to complete in internal testing). Although our initial goal was to create a video of similar length to gameplay, we decided that such a video would contain such a large amount of filler material that it would become ineffective. For the inherent modality of the video format, the team and the instructional designer decided a short video would be the best approach and would lead to the most effective instructional video possible. The final video is 3 minutes long.

\section{VALIDATING THE GAME AND VIDEO}

In order to ensure that the game and video were comparable, we wanted to ensure two premises were satisfied: 1) that the game and the video contained the same content and 2) that the game and the video were of similar quality for their respective modalities. Although we designed the game and the video to satisfy these criteria, our validation would confirm that we had successfully done so with a larger sample external to our team.

\subsection{Validation With Health Experts}

In our first validation study, we recruited 10 health sciences experts from an online freelancing platform. Experts had an average of 8 years $(\mathrm{SD}=8.96)$ of experience in public health. Primary qualifications were medical student (1x), master's degree in a health sciences discipline (5x), practicing physician (3x), and registered nurse (1x). After playing the game and watching the video in a random order, experts filled out a survey. The survey asked participants whether the game and video taught the same material with respect to mask wearing, social distancing, disinfecting workstations, COVID-19 knowledge, and handwashing on a scale from 1:Strongly Disagree to 5:Strongly Agree. Participants then rated the subjective quality of the game and the video each on a scale from 1 to 10. Experts were reimbursed \$20 USD. (See Table 1.) On average, health experts felt that the material was the same across the game and the video (between Agree and Strongly Agree). The game and the video were also rated similarly in terms of their respective quality.

\subsection{Validation With Online Participants}

In our second validation study, we recruited 77 Amazon Mechanical Turk (AMT) participants. All participants were from the U.S. Each participant was compensated \$4 (USD). After an audio check, which confirmed participants had working audio, participants completed the game and video in a random order. Participants then completed the same survey as in the first validation study with health experts. (See Table 1.) Overall, AMT participants agreed that the game and video contained the same instructional material (all dimensions 4.4). AMT workers also rated the quality of the game and the video similarly (both 7.7).

\section{EXPERIMENT 1: CLASSROOM STUDY}

The goal of Experiment 1 was to explore how the game, versus the video, affected students' COVID-19 knowledge, sanitizing selfefficacy, anxiety, attitudes and beliefs, and workstation cleaning behavior. Experiment 1 took place in a classroom lab setting over the course of 3 weeks. The class in which the experiment took place is a large computer programming class, which is a required course for all students in a computing program at a large Midwestern university. There are $\sim 150$ students in this class each semester. The experiment was integrated into regularly scheduled lab sessions for the course from October 19th, 2020 to November 6th, 2020. Incorporating this intervention into an actual scenario and setting in which participants would have to complete weekly lab activities increased the ecological validity of our experiment. Participants first had a pre-test, the intervention (game or video), and a posttest in the first week. In the second and third weeks participants were given a delayed post-test. Additionally, every session was video recorded. See Figure 9 for each participant's workstation and Figure 8 for an overview of Experiment 1 . The experiment was approved by the university's IRB.

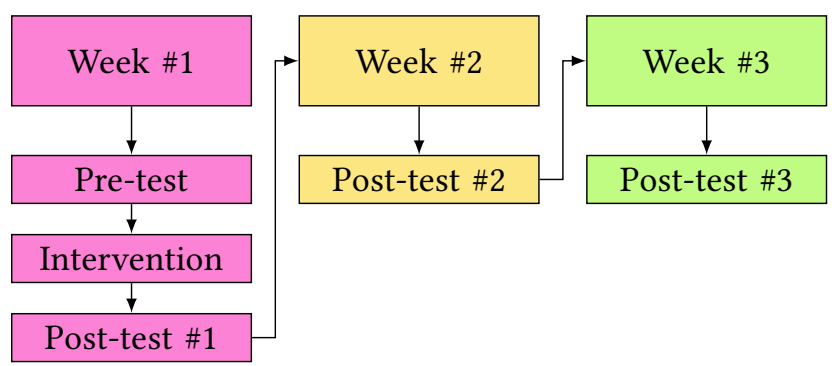

Figure 8: Experiment 1 took place over 3 weeks in a classroom lab setting.

\subsection{Additional Study Context and Recruitment Difficulties During Pandemic}

The study occurred during a time when COVID-19 cases were rising rapidly in the state where this study took place. At the beginning 
Table 1: Summary of answers to the expert and crowdsourced validation studies.

\begin{tabular}{lcccc}
\hline Question & Mean (Expert) & SD (Expert) & Mean (Online) & SD (Online) \\
\hline Same material mask-wearing & 4.8 & 0.42 & 4.43 & 0.52 \\
Same material social distancing & 4.3 & 1.25 & 4.38 & 0.61 \\
Same material disinfecting workstation & 4.5 & 0.71 & 4.44 & 0.60 \\
Same material COVID-19 knowledge & 4.4 & 0.97 & 4.39 & 0.63 \\
Same material handwashing & 4.6 & 0.52 & 4.47 & 0.62 \\
\hline Quality of the game & 8.7 & 1.49 & 7.77 & 2.19 \\
Quality of the video & 9.1 & 0.99 & 7.64 & 2.07 \\
\hline
\end{tabular}
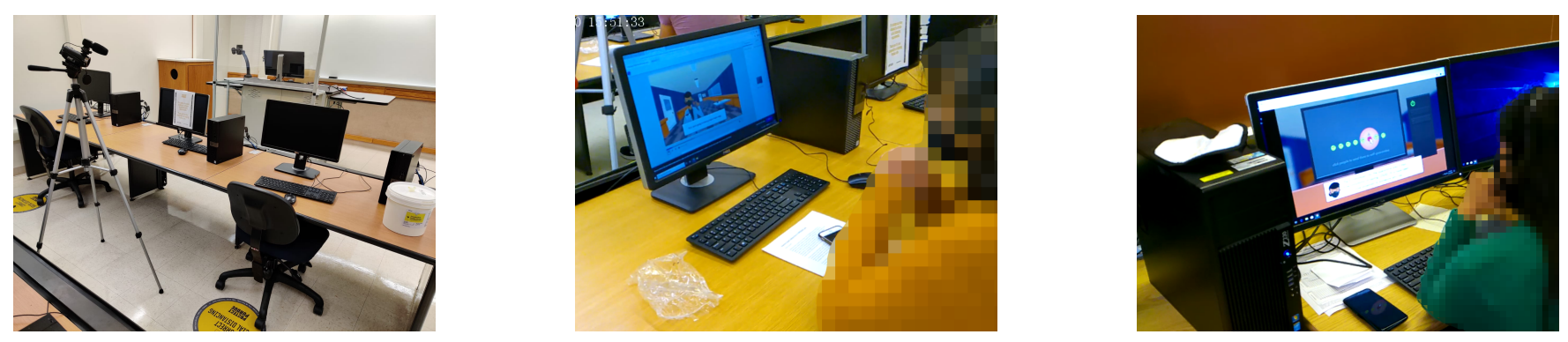

Figure 9: The computer workstation for each student during Experiment 1. During the COVID-19 pandemic, all labs were outfitted with plexiglass barriers, disinfectant wipes, social distancing signs on the floor and on workstations, and signs on each entrance indicating the maximum number of people that can safely be in the room at one time.

of the study, there were 1,583 new COVID-19 cases per day, while at the end there were 4,648 cases $(M=2671)$. Additionally, the university where this study took place instituted a policy in which attendance was not mandatory as a safety precaution, in case students felt unwell but were not officially excused. Because of these circumstances, attendance was much lower than in non-pandemic semesters. Therefore, despite the large number of students enrolled in the class, we were only able to recruit 11 participants to physically attend the study. Finally, we expected that students would come into the experiment with some pre-existing knowledge on COVID-19. In addition to social media and news, the university distributed information through e-mails and video announcements. This included university-wide policies on mask wearing and social distancing, as well as an honor pledge that all students were required to sign before being cleared to return to campus [51].

\subsection{Methodology}

6.2.1 Conditions. The two conditions in this experiment were:

(1) COVID-19 Game
(2) COVID-19 Video

In the game condition, participants play the game (which was described above) from beginning to end. In the video condition, participants watch the video (which was described previously) from beginning to end. All other aspects of the experiment are identical between conditions.

6.2.2 Quantitative and Qualitative Measures. We used measures that assess COVID-19 knowledge, anxiety, attitudes and beliefs, and self-efficacy. Additionally, we use measures to gauge engagement with the game and video. All questionnaires were either formally empirically validated or adapted from publications and official health organizations (e.g., the CDC) and validated by the health sciences experts on the team. Finally, we use recorded video to determine actual cleaning behavior during the labs.

Public Health COVID-19 Knowledge (PHCK): This 5-question measure assessed participants' general COVID-19 knowledge. Questions were equally weighted and worth 1 point each. One question had multiple correct answers, which needed to be selected. For this question, the number of incorrect answers was subtracted from the number of correct answers, with this total then being divided by the total number of correct answers. A higher score on the scale indicates a greater awareness of COVID-19. All questions were created based on official information at the time from the CDC website [17]. See Appendix A.

Shared Workspace COVID-19 Knowledge (SWCK): This 4-question measure assessed COVID-19 knowledge, specifically with respect to shared workspaces. Questions were equally weighted and worth 1 point each. For the open-ended question (List all the frequently touched areas in this lab), for each correct answer listed, the participant earned points equal to a fraction of the total correct answers. A higher score on the scale indicated greater knowledge about safely using shared workspaces. All questions were created based on CDC information [17]. See Appendix B.

COVID-19 Hygienic Workstation Self-Efficacy: This 6-question scale was adapted from the Motivated Strategies for Learning Questionnaire [50]. We use the portion of the scale that refers to selfefficacy beliefs. This scale has been adapted for other tasks (e.g., 
[28]), which we use here to measure self-efficacy in hygienic workstation behavior. All questions are scored from 1:Strongly Disagree to 5:Strongly Agree. A higher score indicated greater self-efficacy. See Appendix C.

COVID-19 Anxiety Scale: This 2-question scale was adapted from the COVID-19 Pandemic Mental Health Questionnaire (CoPaQ) [52]. Questions were adapted to refer to working in a shared workspace. All questions were on a scale of 1:Not At All to 5:Always. A higher score indicates greater anxiety. See Appendix D.

COVID-19 Positive Hygienic Attitude: This 6-question scale was adapted from [7]. All questions were on a scale of 1:Strongly Disagree to 5:Strongly Agree. A higher score indicates stronger positive attitudes. See Appendix E.

Intrinsic Motivation Inventory (IMI): This scale assesses intrinsic motivation relating to a specific activity [47]. We use the following subscales of the IMI: Interest/Enjoyment, Effort/Importance, Pressure/Tension, and Value/Usefulness. For example, "I enjoyed doing this activity very much." All responses range from 1:Not At All True to 7:Very True. Although the IMI contains multiple subscales, the Interest/Enjoyment subscale specifically is known as the subscale that assesses intrinsic motivation [47].

Player Experience Inventory (PXI): This 30-item scale measures player experience at two levels: psychosocial consequences (e.g., immersion) and functional consequences (e.g., progress feedback) [4]. All responses range from -3:Strongly Disagree to 3:Strongly Agree. This scale was administered only to participants in the game condition.

Video Engagement Scale (VES): This 9-question scale measures engagement with a video [65]-e.g., "During viewing I was fully concentrated on the video." Responses range from 1:Completely Disagree to 7:Completely Agree. This scale was administered only to participants in the video condition.

Cleaning Behavior: Finally, we measured actual cleaning behavior during each lab session. Each lab session was video recorded. Two of the authors of this paper (one of whom serves as a teaching assistant in the lab sessions) created a rubric for coding cleaning behavior. This rubric was then iteratively refined through feedback from the two health sciences experts on the team. The final rubric (see Section F) contained five categories: Cleaning before use (the correctness and thoroughness of cleaning prior to the participant sitting down and using a workstation); face mask wearing (the duration for which the participant wore a face mask); absence of eyes, nose, or mouth touching (the absence of unsafe face touching); cleaning after use (the correctness and thoroughness of cleaning after the participant was done using a workstation); and total cleaning time (the combined cleaning before use and cleaning after use time). Each of these categories was coded on a scale from 1:Highly Unsafe Practice to 5:Ideal Practice. Coding was then performed independently by the same authors who created the rubric, blind to the condition. After each iteration of independent coding, the two authors came together to discuss any differences in coding, and the videos were again independently coded. This process was repeated until consensus was reached. In total, 28 videos were coded, with 6 full iterations of coding.

6.2.3 Participants. A total of 11 participants were recruited from the programming class. 9 students attended all 3 weekly sessions.
9 of the 11 participants self-identified as male, and 2 as female. Participants' age was $\mathrm{M}=20.4, \mathrm{SD}=1.6 .10$ of the students were from the Purdue Polytechnic Institute, while 1 was from the College of Liberal Arts. Participants were reimbursed with a \$10 Amazon gift certificate for each of the three experiment sessions they participated in, and they were additionally compensated an additional $\$ 30$ Amazon gift certificate if they completed all three sessions.

6.2.4 Design. A between-subjects design was used. Participants were randomly assigned to either the game or video condition.

6.2.5 Procedure. Participants were recruited by a researcher on the team who was not involved in the programming course as an instructor to avoid undue influence. Students were informed one week ahead of the first scheduled lab session that they would have the option of enrolling in the experiment. When students entered their regularly scheduled lab session, they were given the option of voluntarily enrolling into the study. To avoid undue influence, the professor in charge of the course could not access participant data (i.e., which students enrolled in the study) until final grades were posted for the class. Participants were recruited across 12 different 1hour labs (each has up to 10 students), taught by 3 different teaching assistants.

In the first session, students were first given a consent form. After consenting, participants filled out pre-test questionnaires (PHCK, SWCK, COVID-19 Hygienic Workstation Self-Efficacy, COVID-19 Anxiety, COVID-19 Positive Hygienic Attitude). Next, participants then either played the game or watched the video in their entirety. Students were provided a set of disposable headphones and used those headphones to ensure clear audio. Participants then completed the post-test, which was the same questionnaire as the pre-test. Participants then completed the IMI, then either the PXI (for game condition participants) or the VES (for video condition participants). Finally, participants shared their demographics. The post-test in week 2 contained the COVID-19 Hygienic Workstation Self-Efficacy, COVID-19 Anxiety, and COVID-19 Positive Hygienic Attitude questionnaires. The post-test in week 3 was identical to week 2 but also included the PHCK and SWCK. Each lab session was recorded in its entirety, which gave us the opportunity to observe how students sanitized their workspaces before and after using the workstation.

6.2.6 Analysis. The internal consistency of each self-report measure was analyzed using Cronbach's alpha. When a scale was administered multiple times during the study, the average Cronbach's alpha across the study is reported. In the first session, there were six participants in the game condition and five participants in the video condition. After the first session, two participants from the video condition dropped from the study. For weeks two and three, there were six participants in the game condition and three participants in the video condition. Because of the small sample size, we do not perform statistical analyses and limit our analysis to observation of descriptive differences.

\subsection{Results}

6.3.1 Intrinsic Motivation Inventory. The IMI was administered to the participants once immediately after the intervention. The IMI 


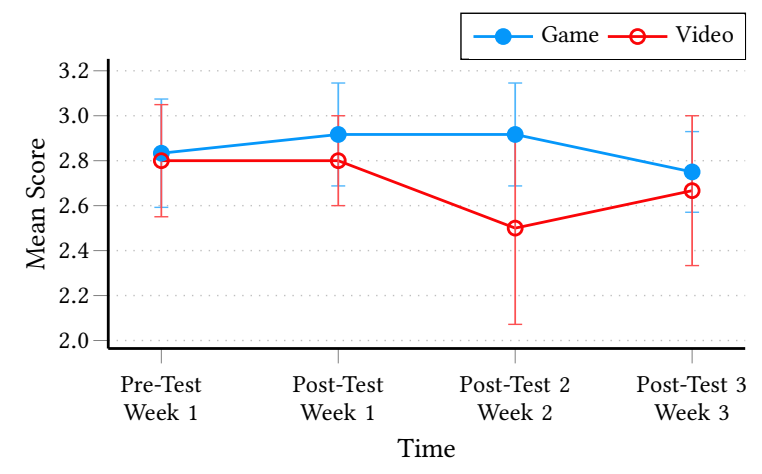

Figure 10: COVID-19 anxiety scores for the in-person study (+/- SEM).

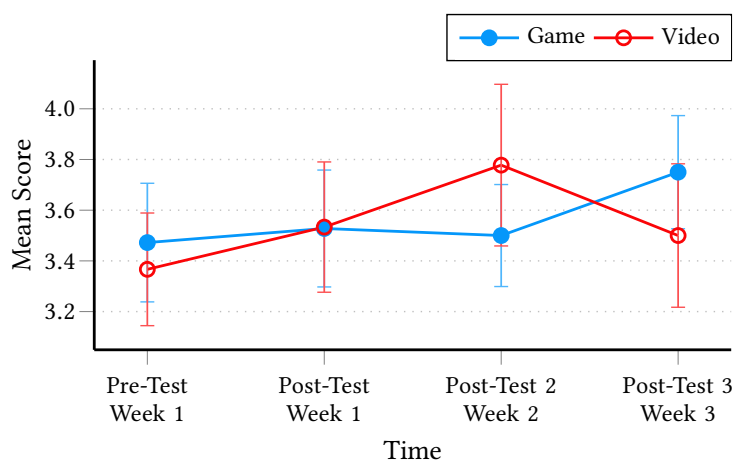

Figure 12: COVID-19 positive hygienic attitude scores for the in-person study (+/- SEM).

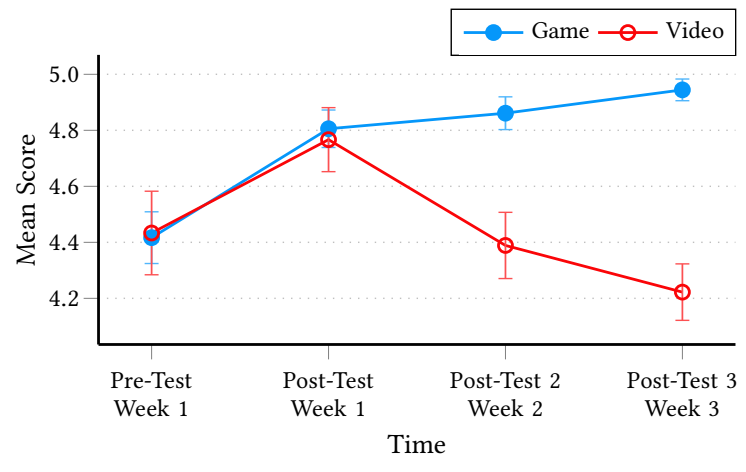

Figure 11: COVID-19 hygienic workstation self-efficacy scores for the in-person study (+/- SEM).

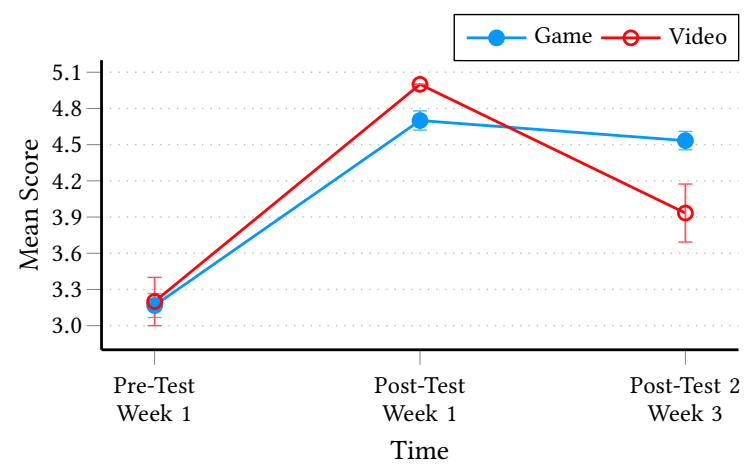

Figure 13: Public Health COVID-19 Knowledge scores for the in-person study (+/- SEM).

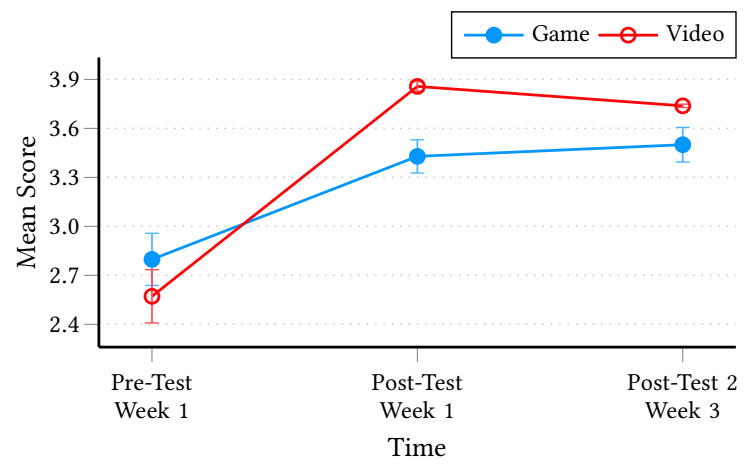

Figure 14: Shared Workspace COVID-19 Knowledge scores for the in-person study (+/- SEM).

was found to be highly reliable across the four subscales: Interest/Enjoyment ( $\alpha=0.90$; Game: $M=4.93, \mathrm{SD}=1.20$; Video: $\mathrm{M}=3.83$, $\mathrm{SD}=0.94)$, Effort/Importance $(\alpha=0.81$; Game: $\mathrm{M}=4.83, \mathrm{SD}=1.45$; Video: $\mathrm{M}=4.08, \mathrm{SD}=1.43)$, Pressure/Tension $(\alpha=0.80$; Game: $\mathrm{M}=1.53$, $\mathrm{SD}=0.58$; Video: $\mathrm{M}=1.28, \mathrm{SD}=0.43)$, and Value/Usefulness $(\alpha=0.84$; Game: $M=5.62, S D=0.94$; Video: $M=5.23, S D=0.31$ ). We observe that the largest difference between conditions was in the subscale of Interest/Enjoyment, the subscale used to assess intrinsic motivation.
6.3.2 PXI and VES. The Player Experience Inventory was administered to the game condition, and the Video Engagement Scale was administered to the video condition, immediately after the intervention. The PXI consisted of 11 subscales of three items each. The VES consisted of 3 subscales of three items each. Table 2 shows the mean scores of PXI along with Cronbach's alpha. With the exception of Mastery $(\alpha=0.37)$ and Autonomy $(\alpha=0.61)$, all subscales reported high reliability. Table 3 shows the participants mean scores of VES 
with Cronbach's alpha. We observe a low alpha for the subscale Attention $(\alpha=0.33)$.

6.3.3 COVID-19 Anxiety. Average Cronbach's alpha for COVID-19 anxiety was $\alpha=0.52$. The minimum mean score was 1 while the maximum was 5 . A higher score indicated a greater level of anxiety of being infected with COVID-19 during workstation use. Participants in general did not differ in their anxiety scores regardless of time. See Figure 10.

6.3.4 COVID-19 Hygienic Workstation Self-Efficacy. Average Cronbach's alpha for COVID-19 hygienic self-efficacy was $\alpha=0.74$. A higher score on this scale indicates greater self-efficacy. Participants scored similarly in the pre-test and improved in their self-efficacy scores in the post-test. The participants belonging to the game condition maintained their scores through week 2 and week 3 of the experiment, while a gradual decline in self-efficacy scores was observed for the participants belonging to the video condition. Figure 11 highlights these trends over the course of the study.

6.3.5 COVID-19 Positive Hygienic Attitude. Average Cronbach's alpha for COVID-19 positive hygienic attitude was $\alpha=0.50$. Participants in both conditions have similar scores at pre-test, and we see a marginal improvement in their mean attitude score over time. Overall the scores of the two conditions do not appear to vary in any specific direction. See Figure 12.

6.3.6 Public Health COVID-19 Knowledge. Participants scored similarly in the pre-test, and both groups improved in their public health COVID-19 knowledge scores in the post-test. Notably, the participants in the video condition scored full points in the post-test given immediately after the intervention but show a sharp decrease in performance in the delayed post-test at the end of week 3 . On the other hand, the performance of the game group was roughly constant for the duration of the study following the intervention. Figure 13 highlights these trends over the course of the study.

6.3.7 Shared Workspace COVID-19 Knowledge (SWCK). Participants scored similarly on shared workspace COVID-19 knowledge in the pre-test and greatly improved in their scores in the post-test. The participants in both conditions maintained their scores in the follow-up test conducted in week 3 . We see that the participants in the video condition consistently performed slightly better than participants in the game condition following the intervention. See Figure 14.

6.3.8 Cleaning Behavior. We wanted to measure if participants spontaneously sanitize their workstation before and after use. Therefore, no prompts to clean the workstation were given at any time throughout the study. A total of 28 videos were qualitatively coded for the analysis. One video from a participant in the video condition in week two was lost due to the camera's view being obstructed. Because of the small sample size, we limit our analysis to describing descriptive results. Figure 15 shows how participants performed in each category over time. In week one, there do not appear to be large differences between the game and video conditions. Both groups have a consistently high face mask wearing score. This is expected since wearing masks is required while indoors inside the university. However, we see low scores on Absence of Face Touching for both groups for the duration of the experiment. In week one, we

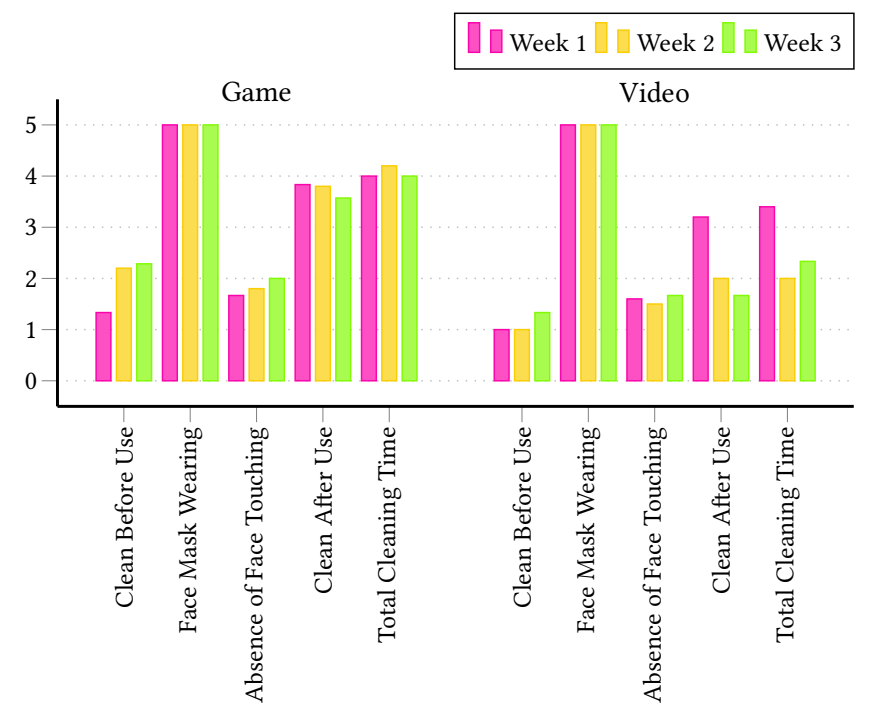

Figure 15: Mean cleaning scores for each construct of the rubric over time.

were interested in whether participants spontaneously sanitized their workstation before they received the intervention. However, both groups scored similarly low on Clean Before Use, indicating that they did not sanitize their workstation before use. After the intervention, participants in the game condition improved slightly in Clean Before Use in week two and three. Other constructs remained mostly similar for the duration of the experiment for participants in the game condition. Interestingly, participants in the video condition appeared to do worse in Clean After Use and Total Cleaning Time in weeks two and three, which may signal that any positive effects from the video on cleaning behavior were brief.

\subsection{Discussion}

Cronbach's alpha values for the IMI, PXI, and VES appear to be highly reliable and consistent across various subscales. Cronbach's alpha for the COVID-19 anxiety, attitudes, and self-efficacy scales varied. For all three scales, we speculate that the low sample size, and sometimes small number of scale items may have created this greater variability in alpha scores. Of particular concern is the highly variable alpha values for the COVID-19 anxiety and attitudes scale. The anxiety scale had only two items, which may have influenced Cronbach's alpha to be lower. We expect that the larger number of participants in the online study will give us a clearer picture of each measure's reliability.

The PHCK and SWCK assessed participants' knowledge regarding COVID-19 hygienic best practices and workspace sanitization. We see that participants in both conditions improve on the knowledge scales, as expected, following the intervention. However, we do see a slightly higher score for the video condition immediately after the intervention. This might be explained by the fact that the information was condensed in a shorter time period for the video (mean completion time for the video was 270 seconds versus 447 seconds for the game), which allowed participants to recall more information (e.g., the forgetting curve which describes the 
decline of memory retention over time [10]). However, by week 3 in the PHCK, this advantage is lost and the game condition actually retains knowledge better than the video condition. In the SWCK on the other hand, both conditions appear to be roughly similar by week 3 . When comparing the cleaning behavior across the two conditions, we see a general, sustained increase in performance of the game group compared to the video group. This result dovetails with the higher self-efficacy for the game group following the intervention. However, given the low sample size of our in-person study, it is difficult to make concrete generalizable claims. Looking at the PXI and VES, we see that most subscales of the PXI are positive, except for Autonomy and Challenge. Therefore, overall the player experience was a positive one on most dimensions, although the game was on the slightly easier side and provided few choices. For the VES, we see a positive score for Attention, but below neutral scores for Emotions and Going Into a Narrative World. This made sense since the video was mainly informational and not a story.

\section{EXPERIMENT 2}

Our second study aimed at obtaining a larger sample size for obtaining more statistically relevant results. Study two is similar to study one; however, we decided on a single delayed post-test after 2 weeks. Because it is an online study, we did not capture actual cleaning behavior during the study. We used Amazon Mechanical Turk (AMT), which has been shown to be reliable for running research studies [46]. Although the game takes place in a university setting, the concepts taught in the game are generally relevant outside of a university setting as well. See Figure 16 .

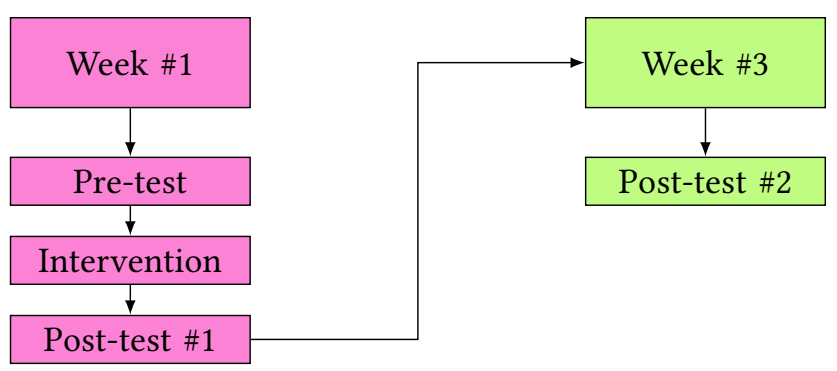

Figure 16: Experiment 2 took place over 3 weeks on Amazon Mechanical Turk.

\subsection{Methodology}

7.1.1 Conditions. Conditions are identical to the conditions from Experiment 1:

(1) COVID-19 Game

(2) COVID-19 Video

7.1.2 Measures. Measures are identical to the measures from Experiment 1, except cleaning behavior is no longer assessed. Slight adaptations were made to the questions to make them no longer targeted towards a specific computer lab. For example, the SWCK question "List all the frequently touched areas in this lab" was changed to "List all the frequently touched areas in a shared computer workstation found in a public library or a university."
7.1.3 Participants. We initially recruited 475 participants. For the post-test, which occurred two weeks later, we were able to retain 396 participants. In cleaning the data, we first excluded participants who did not correctly complete the intervention $(\mathrm{N}=113)$. For example, participants who completed watching the video or playing the game in an impossibly fast amount of time. In the game condition, $\mathrm{N}=63$ participants were excluded in this way. In the video condition, $\mathrm{N}=50$ participants were excluded in this way. For the analyses in this paper, we keep only the participants who were present in both the first session in week one and in the second session in week three $(\mathrm{N}=305)$. This final set of participants contained 138 participants in the game condition and 167 participants in the video condition. The average age of participants was $M=39.3$ $(\mathrm{SD}=11.4)$, with the youngest participant aged 20 and the oldest aged 74 . The sample had 188 participants who self-identified as male ( $62 \%), 115$ who self-identified as female ( $38 \%)$, and 2 who specified a non-binary gender. The majority of the sample selfidentified as White $(\mathrm{N}=241)$, with the second largest racial/ethnic category being Black or African American $(\mathrm{N}=34)$. See Table 4 for a complete breakdown. The majority of participants specified a highest educational level of bachelor's degree $(\mathrm{N}=158)$. The highest number of participants specified a household income of $\$ 37,500$ $\$ 49,999(\mathrm{~N}=60)$. See Tables 5 and 6. Each participant spent roughly one hour in total in completing both sessions. Each participant was paid \$10 (USD) total, split equally between each session. All participants were from the U.S.

7.1.4 Design. A between-subjects design was used. Participants were randomly assigned to either the game or video condition.

7.1.5 Procedure. In the first session, participants first filled out an Institutional Review Board (IRB) approved consent form. After providing consent, participants filled out pre-test questionnaires (PHCK, SWCK, COVID-19 Hygienic Workstation Self-Efficacy, COVID19 Anxiety Scale, COVID-19 Positive Hygienic Attitudes). Next, participants then either played the game or watched the video in their entirety. Participants then completed the post-test, which was the same questionnaire as the pre-test. The participants additionally completed the IMI, then either the PXI (for game condition participants) or the VES (for video condition participants). Finally, participants shared their demographics. The post-test in week three consisted of the same set of questionnaires as in the pre-test.

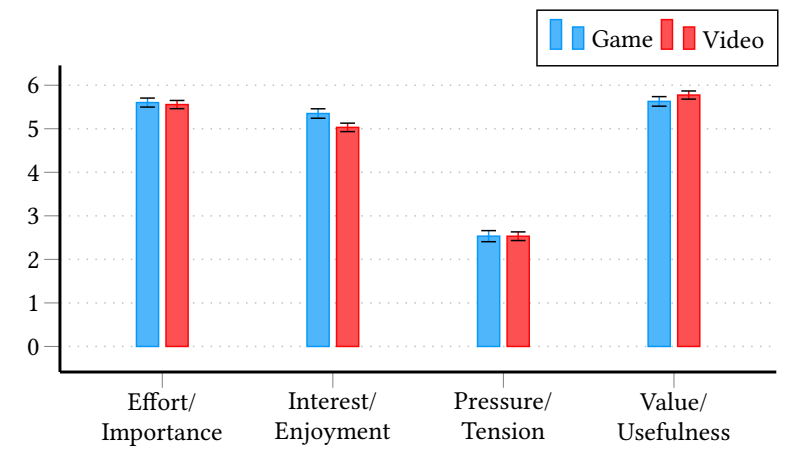

Figure 17: IMI scores for the online study (+/- SEM). 


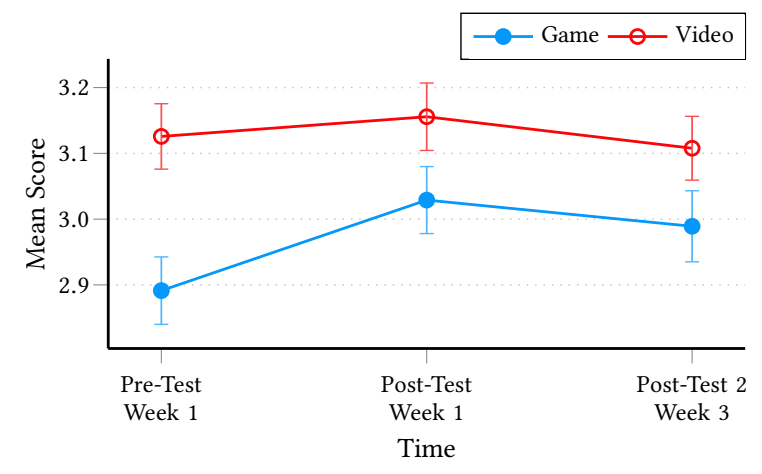

Figure 18: COVID-19 anxiety scores for the online study (+/- SEM).

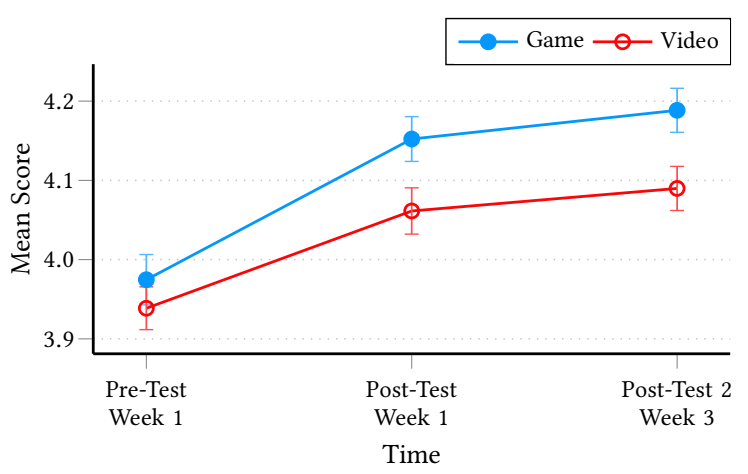

Figure 20: COVID-19 positive hygienic attitude scores for the online study (+/- SEM).

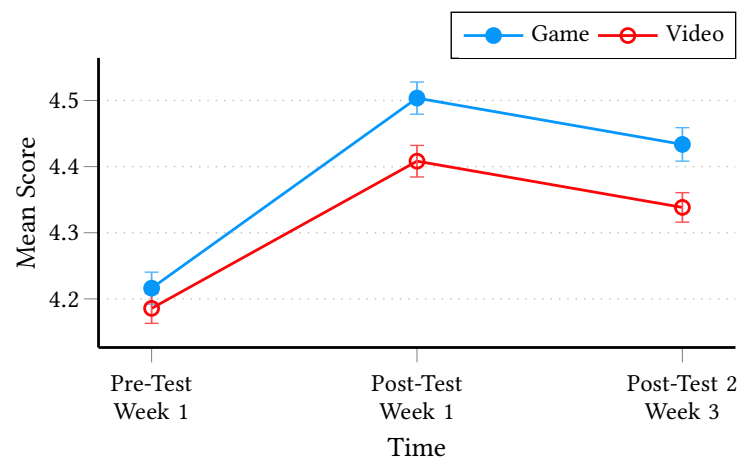

Figure 19: COVID-19 hygienic self-efficacy scores for the online study (+/- SEM).

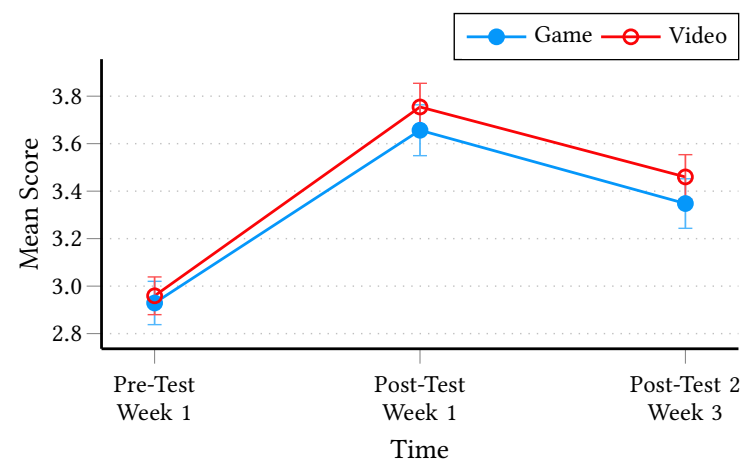

Figure 21: Public Health Knowledge COVID-19 scores for the online study (+/- SEM).

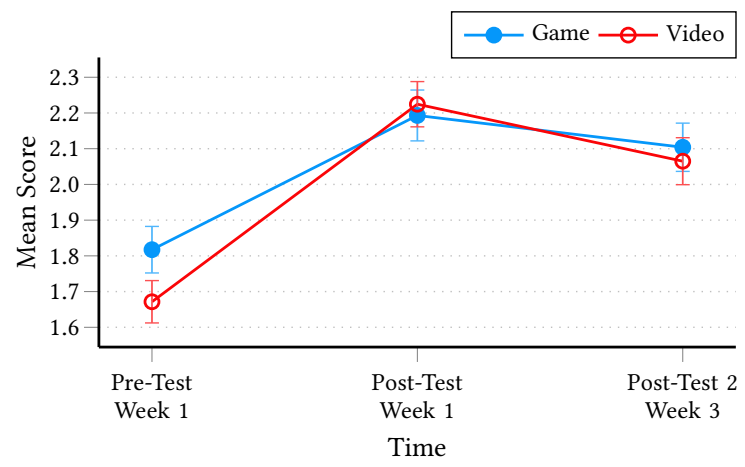

Figure 22: Shared Workspace COVID-19 Knowledge scores for the online study (+/- SEM).

7.1.6 Analysis. To ensure that each questionnaire had good reliability, we conducted Chronbach's Alpha analysis each time the questionnaires were administered to the participants. We then proceed with a repeated measures ANOVA if the questionnaire was found to be reliable $(\alpha \sim 0.7)$. We use two-way repeated measures ANOVA, where the condition is a between-subject factor and time is a within-subject factor. The dependent variables were the scores on each questionnaire. In the case where the questionnaire is only administered a single time (the IMI), we use independent t-tests to compare subscale scores between conditions directly. Prior to running the ANOVA, we checked both the assumption of homogeneity of variance and covariance using Levene's Test of Equality of Error Variance and Box's Test of Equality of Covariance Matrices $(p>0.001)$. These assumptions were met by our data. However, the data of hygienic self-efficacy violated the assumption of equal covariances (Box's Test, $\mathrm{p}=0.001$ ). Based on [59], if group sizes are 
more than 30, then ANOVA is robust to violations of homogeneity of variance-covariance matrices assumptions.

\subsection{Results}

7.2.1 Missingness Analysis. To ensure that the participants who dropped out between week one and week three did not skew our final results in a significant way, we conducted a missingness analysis. We ran a logistic regression with the a dummy dependent variable missing that took a binary value ( 1 for missing and 0 for present), then age (numerical), household income (categorical with nine levels), gender (categorical with three levels), race (categorical with eleven levels), highest education attained (categorical with five levels), and condition (dichotomous) as explanatory variables. We found a significant effect of age $(\beta=-0.04, \mathrm{SE}=0.016, z=2.44$, $p=0.01)$. The mean age of the sample without the missing data was marginally greater $(M=39.31, S D=11.43)$ compared to the sample with the missing values $(M=38.67, S D=11.34)$, suggesting that younger participants were more likely to drop out of the study. None of the other variables reached significance (see Table 9 for the full regression results). We then conducted an independent t-test to see if age differs significantly between the two conditions. We found no significant difference in age between the two conditions, $t(352.24)=0.42, p=0.67$. Because the missingness does not appear to be a result of the conditions themselves, we proceeded with our original analysis plan limited to participants who completed both sessions.

RQ1: How does a COVID-19 game compare against a video in generating intrinsic motivation?

Participants in the game condition rated the task as more intrinsically motivating than participants in the video condition.

The Intrinsic Motivation Inventory was administered to all participants once after the intervention. The IMI was found to be highly reliable across the four subscales: Interest/Enjoyment $(\alpha=0.86)$, Effort/Importance $(\alpha=0.78)$, Pressure/Tension $(\alpha=0.81)$, and Value/ Usefulness $(\alpha=0.94)$. We conducted independent samples t-tests on each subscale across the two conditions. The participants in the game condition reported significantly higher scores on the Interest/Enjoyment subscale $(\mathrm{M}=5.35, \mathrm{SD}=1.27)$ compared to participants in the video condition $(M=5.03, \mathrm{SD}=1.25), t(290.6)=2.18$, $p=0.02, d=0.25$. The Interest/Enjoyment subscale is considered to be the subscale that measures intrinsic motivation [47]. Therefore, participants in the game condition reported being more intrinsically motivated than participants in the video condition. No other subscale reached significance $(p>0.3)$. See Figure 17 .

RQ2: How does a COVID-19 game compare against a video in affecting COVID-19 anxiety?

There were no differences in COVID-19 anxiety between conditions or across time.

The anxiety scale was found to be highly reliable (average Chronbach's $\alpha=0.81$ ). The COVID-19 anxiety scale was administered to the participants three times (pre-test, post-test, and delayed post-test) We conducted a two-way repeated measures ANOVA to understand the effect of intervention on anxiety scores over time. There was no significant effect of time, Wilks' $\lambda=0.98, \mathrm{~F}(2,302)=2.56, p=0.175$, or experimental condition, $\mathrm{F}(1,303)=1.94, p=0.16$. This result suggests that neither condition nor time had an effect on anxiety scores. See Figure 18.

RQ3: How does a COVID-19 game compare against a video in affecting hygienic self-efficacy?

Participants in both game and video conditions improved in hygienic self-efficacy. There was no significant difference between conditions. Average Cronbach's alpha for COVID-19 hygienic self-efficacy was $\alpha=0.85$, indicating high reliability. The self-efficacy questionnaire was administered to participants three times (pre-test, post-test, and delayed post-test). We conducted a two-way repeated measures ANOVA with the self-efficacy scores as the dependent variable. We found a significant effect of time, Wilks' $\lambda=0.749, \mathrm{~F}(2,302)=50.75$, $p<0.001, \eta_{p}^{2}=0.25$. There was no significant effect of condition, $\mathrm{F}(1,303)=2.274, p=0.13$. This suggests that the game and video both positively affected self-efficacy scores. We computed paired sample t-tests to compare the difference in self-efficacy scores over time. The first paired sample t-test indicated a significant difference between the mean scores for the pre-test $(M=25.20, S D=3.00)$ and post-test $(M=26.71, S D=3.11), p<0.001$. The second paired sample t-test indicated a significant difference between the mean scores for the post-test and the delayed post-test $(M=26.29, \mathrm{SD}=3.05), p<0.001$. The third paired sample t-test indicated a significant difference between the mean scores for the delayed post-test and the pre-test, $p<0.001$. All post-hoc tests were Bonferroni corrected. See Figure 19.

RQ4: How does a COVID-19 game compare against a video in affecting positive hygienic attitude?

Participants in both game and video conditions improved in positive hygienic attitude. There was no significant difference between conditions.

The positive hygienic attitude scale was administered to participants three times (pre-test, post-test, and delayed post-test). Due to an initially low reliability ( $\alpha=0.45$ in the pre-test and $\alpha=0.52$ in the post-test), we dropped items 2 and 4 from the attitude questionnaire. After dropping these items, the scale had an acceptable reliability (average Cronbach's alpha was $\alpha=0.65$ ). We conducted a two-way repeated measures ANOVA with the attitude scores as the dependent variable. We found a significant effect of time, Wilks' $\lambda=0.86, \mathrm{~F}(2,302)=24.56, p<0.001, \eta_{p}^{2}=0.14$. There was no significant effect of condition, $\mathrm{F}(1,303)=1.427, p=0.23$. This suggests that both the game and video positively affected attitude scores. We computed paired sample t-tests to compare the difference in attitude scores over time. The first paired sample t-test indicated a significant difference between the mean scores for the pre-test $(M=15.82$, $\mathrm{SD}=2.41)$ and post-test $(\mathrm{M}=16.41, \mathrm{SD}=2.48), p<0.001$. There was no significant difference between the immediate post-test and the delayed post-test $(\mathrm{p}=0.78)$. The third paired sample t-test indicated a significant difference between the mean scores for the delayed post-test $(\mathrm{M}=16.54, \mathrm{SD}=2.39)$ and the pre-test, $p<0.001$. All post-hoc tests were Bonferroni corrected. See Figure 20.

RQ5: How does a COVID-19 game compare against a video in teaching COVID-19 knowledge? 
Participants in both game and video conditions improved in COVID19 knowledge. There was no significant difference between conditions. The PHCK and SWCK scales were administered to the participants three times (pre-test, post-test, and delayed post-test). We conducted a two-way repeated measures ANOVA with PHCK score as the dependent variable. We found a significant effect of time, Wilks' $\lambda=0.577, \mathrm{~F}(2,302)=110.86, p<0.001, \eta_{p}^{2}=0.42$. We did not observe a significant difference between conditions, $F(1,303)=0.46$, $p=0.49$. This suggests that both the game and video positively affected PHCK scores. We computed paired sample t-tests to compare the difference in PHCK scores across time. The first paired sample $\mathrm{t}$-test indicated a significant difference between the mean scores for the pre-test $(\mathrm{M}=2.94, \mathrm{SD}=1.04)$ and post-test $(\mathrm{M}=3.71, \mathrm{SD}=1.27)$, $p<0.001$. The second paired sample t-test indicated a significant difference between the mean scores for the post-test and the delayed post-test $(\mathrm{M}=3.40, \mathrm{SD}=1.21), p<0.001$. The third paired sample t-test indicated a significant difference between the mean scores for the delayed post-test and pre-test, $p<0.001$. All post-hoc tests were Bonferroni corrected. We conducted a two-way repeated measures ANOVA with SWCK score as the dependent variable. We found a significant effect of time, Wilks' $\lambda=0.68, F(2,302)=69.35, p<0.001$, $\eta_{p}^{2}=0.42$. We did not observe a significant difference between conditions, $\mathrm{F}(1,303)=0.40, p=0.52$. This suggests that both the game and video positively affected SWCK scores. We computed paired sample t-tests to compare the difference in SWCK scores across time. The first paired sample t-test indicated a significant difference between the mean scores for the pre-test $(M=1.73, \mathrm{SD}=0.76)$ and post-test $(M=2.21, S D=0.82), p<0.001$. The second paired sample t-test indicated a significant difference between the mean scores for the post-test and delayed post-test $(\mathrm{M}=2.08, \mathrm{SD}=0.82), p<0.001$. The third paired sample t-test indicated a significant difference between the mean scores for the delayed post-test and pre-test, $p=0.005$. All post-hoc tests were Bonferroni corrected. See Figures 21 and 22.

7.2.2 PXI and VES. The PXI consisted of 11 subscales (3 items each) and was only administered to the game condition. All subscales reported high reliability. See Table 7 for PXI mean scores and reliability alphas. The VES consisted of 3 subscales ( 3 items each) and was only administered to the video condition. We observe a low reliability value (0.56) for the subscale Attention. See Table 8.

\subsection{Discussion}

A general trend observed across scales-hygienic self-efficacy, positive hygienic attitude, PHCK, and SWCK-was a significant improvement in scores following the intervention. Participants in both conditions improved on their performance on these scales following the intervention. Importantly, this improvement was sustained over a longer period of time, as indicated by the significantly greater scores on the delayed post-test compared to the pre-test. The participants in the game condition showed a higher level of intrinsic motivation compared to the participants in the video condition. Neither condition affected the COVID-19 anxiety scores of participants.

Cronbach's alpha values for IMI, PXI, and VES were observed to be highly reliable and consistent across various subscales. For the psychometric scales, we found an improvement in reliability scores compared to Experiment 1 . The attitude scale had an initial low reliability, but after dropping two items showed an acceptable level of reliability. Looking at the PXI and VES, we observe positive scores on all subscales of the PXI, indicating an overall positive player experience. The scores for the subscale Autonomy and Challenge hovered around zero, which mirrors the results from the in-person study. For the VES, we see a higher score for Attention and a slightly lower score for Emotion and Going into a Narrative World, also mirroring the results from the in-person study.

\section{FOLLOW-UP GAME FOR PUBLIC DISSEMINATION}

In addition to the game described in this paper, we have developed a follow-up game that is intended for wider distribution to the public and can run on both mobile and desktop devices (see footnote ${ }^{45}$ ). The game was developed iteratively by our team with the same approach as the original game. However, the setting of the game is slightly different to resemble a non-university setting. For example, the player begins in their home and must find different hygienic items, run to catch the bus while social distancing, make a seating arrangement on the bus, and sanitize their office. Although many of the challenges are similar to the original game, we added a score feature in which the player is able to collect coins from completing challenges and which are hidden on the screen to increase the replayability of the game. There is also an inventory in which the player stores their important hygienic items (e.g., hand sanitizer). We hope to use this game to conduct further studies in this domain.

\section{DISCUSSION}

We observed a few common trends across both experiments. Participants show a consistent increase in COVID-19 hygienic self-efficacy, COVID-19 knowledge, and COVID-19 positive hygienic attitude in both the game and the video conditions. These increases were sustained longitudinally over the 3 weeks studied. Participants in the game condition experienced higher intrinsic motivation. Neither condition affected COVID-19 anxiety scores.

However, some differences between the two experiments should be noted. Cronbach's alpha values in study one were lower compared to the values in study two. We mainly attribute this difference to the smaller sample size in the first study. SWCK and hygienic self-efficacy scores differed across the two experiments. Comparing the SWCK scores, we observed a higher score for the in-person participants compared to the online participants across both pre-test and post-tests. The in-person students likely had better pre-existing training, found the instruction more relevant to their current situation, and may have paid more attention. Additionally, the majority of the in-person participants ( 93\%) cleaned their shared workstation each week during the study. This reinforcement of the material (i.e., active learning [40] and practice [15]) being taught in the game and video could also have contributed to higher COVID-19 knowledge and hygienic self-efficacy scores. Online participants in the video condition, however, scored higher on the subscales Emotion and Going into the Narrative World of the VES, compared to the in-person participants in the video condition. This suggests that

\footnotetext{
${ }^{4}$ Video of Gameplay (workplace version): https://youtu.be/ppLVjSbIoB4 ${ }^{5}$ Playable Game (workplace version): http://web.ics.purdue.edu/ kaod/ COVIDGameWorkplace
} 
online participants had greater engagement with the video content compared to in-person participants. Online participants may have appreciated the conciseness of the video.

Our results highlight that a digital game intervention can improve knowledge, attitudes, and self-efficacy over a longer period of time. Digital interventions such as these are scalable and can be quickly created and deployed. This is crucial given the changing nature of the pandemic (e.g., the first COVID-19 variant of concern from December 2020 [18]), and where new information may be needed to supplant previous guidelines (e.g., scientific evidence of airborne transmission of COVID-19). The COVID-19 pandemic makes delivering in-person interventions difficult, and therefore, digital interventions such as the one described here can be an effective alternative. While there has been a rise in digital interventions created, many such interventions lack empirical testing [55]. We have described one of the first COVID-19 games that combines teaching both personal and environmental hygienic practices and have performed two evaluation studies during the pandemic.

\section{LIMITATIONS}

There are a few notable limitations in our studies. Firstly, both the in-person and online studies had trade-offs. While the in-person study was highly ecologically valid with the study occurring in a live programming class during the semester, it had a small sample size due to low attendance during the pandemic. While the online study had a large sample size, it was less ecologically valid since the learning material may have been less relevant to the participants (e.g., participants who work from home and do not need to sanitize public workstations). Moreover, we could not measure the actual cleaning behavior of online study participants. That being said, because we were able to run both experiments, we were able to find commonalities between the two experiments' results and derive slightly more robust conclusions.

Many of the scales used in the study were adapted or developed from previous existing scales. Although these scales show good reliability, they may not be as robust as other established scales. That being said, a rapidly changing pandemic makes using robust psychometrically validated scales difficult, since such scales take time to develop and empirically test. Very few inventories exist that assess COVID-19-specific measures in people during the pandemic. More research is needed to create inventories that can be easily adapted to different scenarios (e.g., the IMI).

We did not find a significant difference in participants' cleaning behavior scores over time. Future studies should consider measuring cleaning behavior with a greater sample size to make a more robust conclusion. Another significant limitation is the Hawthorne effect. Although we studied actual cleaning behavior in the in-person study, their behavior may have been influenced by the awareness that they were being watched (e.g., through the video recording device). Because of the limitations including the small sample size, we reported the in-person results descriptively rather than using statistical tests.

\section{CONCLUSION}

COVID-19 has caused irreparable social and economic damage. In this paper, we describe the creation of a game called Fighting COVID19 at Purdue University with professional game designers, health experts, and education technology designers. We then performed both an in-person $(\mathrm{N}=11)$ and online study $(\mathrm{N}=475)$ comparing the game to a comparable video. The studies each lasted three weeks. We find that the game is more intrinsically motivating than the video. Participants in both the game and video conditions have a sustained rise in COVID-19 hygienic self-efficacy, positive hygienic attitudes, and knowledge. Neither of the two conditions had any change in COVID-19 anxiety. Therefore, game-based learning can be an effective approach for teaching COVID-19 hygienic knowledge, for improving COVID-19 hygienic self-efficacy, and for fostering COVID-19 hygienic positive attitudes, and is more intrinsically motivating than video-based learning. Fighting COVID-19 at Purdue University is the first game, to the best of our knowledge, that takes a multi-faceted approach through teaching both personal and environmental hygienic best practices.

\section{ACKNOWLEDGMENTS}

This work was supported by a Protect Purdue Faculty Grant.

\section{REFERENCES}

[1] [n.d.]. Hygiene | WHO | Regional Office for Africa. https://www.afro.who.int/ health-topics/hygiene

[2] [n.d.]. Important Store Info. https://corporate.walmart.com/important-store-info

[3] [n.d.]. Protect Purdue Plan. https://protect.purdue.edu/plan/

[4] Vero Vanden Abeele, Katta Spiel, Lennart Nacke, Daniel Johnson, and Kathrin Gerling. 2020. Development and validation of the player experience inventory: A scale to measure player experiences at the level of functional and psychosocial consequences. International fournal of Human Computer Studies 135, January 2019 (2020), 102370. https://doi.org/10.1016/j.ijhcs.2019.102370

[5] Ghaleb Adwan, Yousef Salama, and Nael Hasan. 2016. Microbial Contamination of Environmental Surfaces in An-Najah National University Setting. Fournal of Scientific Research and Reports (2016). https://doi.org/10.9734/jsrr/2016/23098

[6] Ghaleb Adwan, Yousef Salama, and Nael Abu Hasan. 2016. Microbial contamination of environmental surfaces in An-Najah National University setting. Fournal of Scientific Research and Reports (2016), 1-9.

[7] Mohammed K. Al-Hanawi, Khadijah Angawi, Noor Alshareef, Ameerah M.N. Qattan, Hoda Z. Helmy, Yasmin Abudawood, Mohammed Alqurashi, Waleed M. Kattan, Nasser Akeil Kadasah, Gowokani Chijere Chirwa, and Omar Alsharqi. 2020. Knowledge, Attitude and Practice Toward COVID-19 Among the Public in the Kingdom of Saudi Arabia: A Cross-Sectional Study. Frontiers in Public Health (2020). https://doi.org/10.3389/fpubh.2020.00217

[8] Benedetta Allegranzi and Didier Pittet. 2009. Role of hand hygiene in healthcareassociated infection prevention. Journal of hospital infection 73, 4 (2009), 305-315.

[9] Marcella Alsan, Stefanie Stantcheva, David Yang, and David Cutler. 2020. Disparities in coronavirus 2019 reported incidence, knowledge, and behavior among US adults. FAMA network open 3, 6 (2020), e2012403-e2012403.

[10] Lee Averell and Andrew Heathcote. 2011. The form of the forgetting curve and the fate of memories. Fournal of Mathematical Psychology 55, 1 (2011), 25-35.

[11] Philip Ball and Amy Maxmen. 2020. The epic battle against coronavirus misinformation and conspiracy theories. Nature (2020), 371-374.

[12] Tom Baranowski, Richard Buday, Debbe I. Thompson, and Janice Baranowski. 2008. Playing for Real. Video Games and Stories for Health-Related Behavior Change. American fournal of Preventive Medicine 34, 1 (2008). https://doi.org/10. 1016/j.amepre.2007.09.027

[13] Carol W Bassim, Gretchen Gibson, Timothy Ward, Brian M Paphides, and Donald J DeNucci. 2008. Modification of the risk of mortality from pneumonia with oral hygiene care. Journal of the American Geriatrics Society 56, 9 (2008), 1601-1607.

[14] Sally F. Bloomfield, Allison E. Aiello, Barry Cookson, Carol O'Boyle, and Elaine L. Larson. 2007. The effectiveness of hand hygiene procedures in reducing the risks of infections in home and community settings including handwashing and alcohol-based hand sanitizers. American fournal of Infection Control 35, 10 SUPPL. 1 (2007). https://doi.org/10.1016/j.ajic.2007.07.001

[15] Bruce K Bromage and Richard E Mayer. 1986. Quantitative and qualitative effects of repetition on learning from technical text. Fournal of Educational Psychology 78, 4 (1986), 271.

[16] Ellen Brox, L. Fernandez-Luque, and T. Tøllefsen. 2011. Healthy gaming - Video game design to promote health. Applied Clinical Informatics 2, 2 (2011), 128-142. https://doi.org/10.4338/ACI-2010-10-R-0060 
[17] CDC. 2020. Coronavirus (COVID-19). https://www.cdc.gov/coronavirus/2019ncov/index.html

[18] CDC. 2020. Interim: Implications of the Emerging SARS-CoV-2 Variant VOC 202012/01. https://www.cdc.gov/coronavirus/2019-ncov/more/scientific-briefemerging-variant.html

[19] CDC. 2020. Long-Term Effects of COVID-19. https://www.cdc.gov/coronavirus/ 2019-ncov/long-term-effects.html

[20] CDC. 2020. When and How to Wash Your Hands. https://www.cdc.gov/ handwashing/when-how-handwashing.html

[21] CDC and Epa. 2020. Guidance for Cleaning and Disinfecting Public Spaces, Work places, Businesses, Schools, and Homes. Technical Report.

[22] Anyim Chukwudi. 2013. Bacteriological Examination of Computer Keyboards and Mouse Devices and their Susceptibility Patterns to Disinfectants. American Journal of Bioscience and Bioengineering (2013). https://doi.org/10.11648/j.bio. 20130103.11

[23] Anyim Chukwudi, CL Donatus, and CO Eucharia. 2013. Bacteriological examination of Computer keyboards and mouse devices and their susceptibility patterns to disinfectants. American fournal of Bioscience and Bioengineering 1, 3 (2013), $36-43$.

[24] Lauren Clack, Christian Hirt, Marcel Wenger, Dirk Saleschus, Andreas Kunz, and Hugo Sax. 2019. VIRTUE - A virtual reality trainer for hand hygiene. 2018 9th International Conference on Information, Intelligence, Systems and Applications, IISA 2018 (2019). https://doi.org/10.1109/IISA.2018.8633588

[25] R Dennis Cook and Sanford Weisberg. 2009. Applied regression including computing and graphics. Vol. 488. John Wiley \& Sons.

[26] Val Curtis, Adam Biran, Katie Deverell, Clarissa Hughes, Kate Bellamy, and Bo Drasar. 2003. Hygiene in the home: relating bugs and behaviour. Social science \& medicine 57, 4 (2003), 657-672.

[27] Nese Sevim Cirak and Osman Erol. 2020. What are the factors that affect the motivation of digital gamers? Participatory Educational Research 7, 1 (2020), 184-200. https://doi.org/10.17275/per.20.11.7.1

[28] Jennifer DeBoer, Casey Haney, S. Zahra Atiq, Casey Smith, and David Cox 2019. Hands-on engagement online: using a randomised control trial to estimate the impact of an at-home lab kit on student attitudes and achievement in a MOOC. European fournal of Engineering Education (2019). https://doi.org/10. 1080/03043797.2017.1378170

[29] David Farrell, Patty Kostkova, Julius Weinberg, Lisa Lazareck, Dasun Weerasinghe, Donna M Lecky, and Cliodna AM McNulty. 2011. Computer games to teach hygiene: an evaluation of the e-Bug junior game. Fournal of antimicrobial chemotherapy 66, suppl_5 (2011), v39-v44.

[30] Natalie Gold, Xiao-Yang Hu, Sarah Denford, Ru-Yu Xia, Lauren Towler, Julia Groot, Rachel Gledhill, Merlin Willcox, Ben Ainsworth, Sascha Miller, et al. 2020 Effectiveness of digital interventions to improve household and community infection prevention and control behaviours and to reduce incidence of respiratory and/or gastro-intestinal infections: A rapid systematic review. medRxiv (2020).

[31] Zhen-Dong Guo, Zhong-Yi Wang, Shou-Feng Zhang, Xiao Li, Lin Li, Chao Li, Yan Cui, Rui-Bin Fu, Yun-Zhu Dong, Xiang-Yang Chi, et al. 2020. Aerosol and surface distribution of severe acute respiratory syndrome coronavirus 2 in hospital wards, Wuhan, China, 2020. Emerg Infect Dis 26, 7 (2020), 10-3201.

[32] Zhen Dong Guo, Zhong Yi Wang, Shou Feng Zhang, Xiao Li, Lin Li, Chao Li, Yan Cui, Rui Bin Fu, Yun Zhu Dong, Xiang Yang Chi, Meng Yao Zhang, Kun Liu, Kun Liu, Cheng Cao, Bin Liu, Ke Zhang, Yu Wei Gao, Bing Lu, and Wei Chen. 2020. Aerosol and Surface Distribution of Severe Acute Respiratory Syndrome Coronavirus 2 in Hospital Wards, Wuhan, China, 2020. Emerging Infectious Diseases (2020). https://doi.org/10.3201/eid2607.200885

[33] A. Higgins and M. M. Hannan. 2013. Improved hand hygiene technique and compliance in healthcare workers using gaming technology. Fournal of Hospital Infection 84, 1 (2013), 32-37. https://doi.org/10.1016/j.jhin.2013.02.004

[34] Patricia P Horan, Margaret C Yarborough, Garry Besigel, and Debbie R Carlson. 1990. Computer-assisted self-control of diabetes by adolescents. The Diabetes Educator 16, 3 (1990), 205-211.

[35] Stefan Jooss. 2017. CatCare: Designing a serious game to foster hand hygiene compliance in health care facilities. (2017).

[36] Ya Shu Kang and Yao Jen Chang. 2019. Using a motion-controlled game to teach four elementary school children with intellectual disabilities to improve hand hygiene. Fournal of Applied Research in Intellectual Disabilities 32, 4 (2019), 942-951. https://doi.org/10.1111/jar.12587

[37] Dominic Kao and D. Fox Harrell. 2017. MazeStar: A platform for studying virtual identity and computer science education. ACM International Conference Proceeding Series Part F1301 (2017), 1-6. https://doi.org/10.1145/3102071.3116221

[38] Pamela M Kato, Steve W Cole, Andrew S Bradlyn, and Brad H Pollock. 2008. A video game improves behavioral outcomes in adolescents and young adults with cancer: a randomized trial. Pediatrics 122, 2 (2008), e305-e317.

[39] Ashraf I. Khasawneh, Anas Abu Humeidan, Jomana W. Alsulaiman, Sarah Bloukh, Mohannad Ramadan, Tariq N. Al-Shatanawi, Hasan H. Awad, Waleed Y. Hijazi, Kinda R. Al-Kammash, Nail Obeidat, Tareq Saleh, and Khalid A. Kheirallah. 2020. Medical Students and COVID-19: Knowledge, Attitudes, and Precautionary
Measures. A Descriptive Study From Jordan. Frontiers in Public Health 8, May (2020), 1-9. https://doi.org/10.3389/fpubh.2020.00253

[40] Paul H Kvam. 2000. The effect of active learning methods on student retention in engineering statistics. The American Statistician 54, 2 (2000), 136-140.

[41] Jerika T. Lam, Mary A. Gutierrez, Jeff A. Goad, Larisa Odessky, and Jason Bock. 2019. Use of virtual games for interactive learning in a pharmacy curriculum. Currents in Pharmacy Teaching and Learning 11, 1 (2019), 51-57. https://doi.org/ 10.1016/j.cptl.2018.09.012

[42] Markus Langer, Cornelius J. König, Patrick Gebhard, and Elisabeth André. 2016. Dear Computer, Teach Me Manners: Testing Virtual Employment Interview Training. International fournal of Selection and Assessment 24, 4 (2016), 312-323. https://doi.org/10.1111/ijsa.12150

[43] Debra A Lieberman. 1997. Interactive video games for health promotion: Effects on knowledge, self-efficacy, social support, and health. Health promotion and interactive technology: Theoretical applications and future directions (1997), 103120.

[44] Prof Little, Paul, PhD Stuart, Beth, Prof Hobbs, F D R, Prof Moore, Mike, BA Barnett, Jane, MSc Popoola, Deborah, Karen Middleton, MSc Kelly, Joanne, MSc Mullee, Mark, Prof Raftery, James, PhD Yao, Guiqing, Prof Carman, William, PhD Fleming, Douglas, PhD Stokes-Lampard, Helen, MRCGP Williamson, Ian, PhD Joseph, Judith, PhD Miller, Sascha, and Prof Yardley, Lucy. 2015. An internetdelivered handwashing intervention to modify influenza-like illness and respiratory infection transmission (PRIMIT): a primary care randomised trial. The Lancet (British edition) 386, 10004 (2015), 1631-1639.

[45] Emma Marris. 2020. Millions of students are returning to US universities in a vast unplanned pandemic experiment. Nature 584, 7822 (2020), 510-512.

[46] Winter Mason and Siddharth Suri. 2012. Conducting behavioral research on Amazon's Mechanical Turk. Behavior Research Methods 44, 1 (2012), 1-23. https: //doi.org/10.3758/s13428-011-0124-6 arXiv:/ssrn.com/abstract=1691163 [http:]

[47] Edward McAuley, Terry Duncan, and Vance V Tammen. 1989. Psychometric properties of the Intrinsic Motivation Inventory in a competitive sport setting: A confirmatory factor analysis. Research Quarterly for Exercise and Sport 60, 1 (1989), 48-58.

[48] Marina Papastergiou. 2009. Digital Game-Based Learning in high school Computer Science education: Impact on educational effectiveness and student motivation. Computers and Education 52, 1 (2009), 1-12. https://doi.org/10.1016/j. compedu.2008.06.004

[49] Jocelyn Parong and Richard Mayer. 2018. Learning Science in Immersive Virtual Reality Article in Journal of Educational Psychology. Fournal of Educational Psychology 110, 6 (2018), 785-797. https://doi.org/10.1037/edu0000241

[50] Paul R. Pintrich, David A. F. Smith, Teresa Garcia, and Wilbert J. Mckeachie. 1993. Reliability and Predictive Validity of the Motivated Strategies for Learning Questionnaire (Mslq). Educational and Psychological Measurement (1993). https: //doi.org/10.1177/0013164493053003024

[51] Purdue University. 2020. Protect Purdue Pledge. https://protect.purdue.edu/ pledge/

[52] S. Rek, D. Freeman, M. Reinhard, D. Keeser, and F. Padberg. 2020. The Covid-19 Pandemic Mental Health Questionnaire (CoPaQ): Introducing a comprehensive measure of the psychosocial impact of the coronavirus crisis.

[53] George Stanley Reynolds. 1975. A primer of operant conditioning. Scott Foresman.

[54] Thomas J Sandora, Mei-Chiung Shih, and Donald A Goldmann. 2008. Reducing absenteeism from gastrointestinal and respiratory illness in elementary schoo students: a randomized, controlled trial of an infection-control intervention. Pediatrics 121, 6 (2008), e1555-e1562.

[55] Lamyae Sardi, Ali Idri, and José Luis Fernández-Alemán. 2017. A systematic review of gamification in e-Health. Journal of Biomedical Informatics 71 (2017), 31-48. https://doi.org/10.1016/j.jbi.2017.05.011

[56] Douglas R Southard and Barbara H Southard. 2006. Promoting physical activity in children with MetaKenkoh. Clinical and investigative medicine 29, 5 (2006), 293

[57] Leanne J Staniford and Kelly A Schmidtke. 2020. A systematic review of handhygiene and environmental-disinfection interventions in settings with children. BMC public health 20, 1 (2020), 195.

[58] Mélanie Suppan, Gaud Catho, Tomás Robalo Nunes, Valérie Sauvan, Monique Perez, Christophe Graf, Didier Pittet, Stephan Harbarth, Mohamed Abbas, and Laurent Suppan. 2020. A Serious Game Designed to Promote Safe Behaviors Among Health Care Workers During the COVID-19 Pandemic: Development of "Escape COVID-19". FMIR serious games 8, 4 (2020), e24986.

[59] Barbara G Tabachnick, Linda S Fidell, and Jodie B Ullman. 2007. Using multivariate statistics. Vol. 5. Pearson Boston, MA.

[60] Debbe Thompson, Tom Baranowski, Richard Buday, Janice Baranowski, Victoria Thompson, Russell Jago, and Melissa Juliano Griffith. 2010. Serious video games for health: How behavioral science guided the development of a serious video game. Simulation \& gaming 41, 4 (2010), 587-606.

[61] James B Tidwell, Anila Gopalakrishnan, Stephen Lovelady, Esha Sheth, Arathi Unni, Richard Wright, Shonali Ghosh, and Myriam Sidibe. 2019. Effect of two complementary mass-scale media interventions on handwashing with soap among mothers. fournal of health communication 24, 2 (2019), 203-215. 
[62] Michelle Torok, Jin Han, Simon Baker, Aliza Werner-Seidler, Iana Wong, Mark E. Larsen, and Helen Christensen. 2020. Suicide prevention using self-guided digital interventions: a systematic review and meta-analysis of randomised controlled trials. The Lancet Digital Health 2, 1 (2020), e25-e36. https://doi.org/10.1016/ S2589-7500(19)30199-2

[63] Matti Uhari and MERJA MÖTTÖNEN. 1999. An open randomized controlled trial of infection prevention in child day-care centers. The Pediatric infectious disease journal 18, 8 (1999), 672-677.

[64] Elizabeth Unni, Susan Gabriel, and Rinat Ariely. 2018. A review of the use and effectiveness of digital health technologies in patients with asthma. Annals of Allergy, Asthma \& Immunology 121, 6 (2018), 680-691.

[65] Leonie N.C. Visser, Marij A. Hillen, Mathilde G.E. Verdam, Nadine Bol, Hanneke C.J.M. de Haes, and Ellen M.A. Smets. 2016. Assessing engagement while viewing video vignettes; validation of the Video Engagement Scale (VES). Patient Education and Counseling 99, 2 (2016), 227-235. https://doi.org/10.1016/j.pec. 2015.08.029
[66] Wikipedia. 2020. COVID-19 pandemic data. https://en.wikipedia.org/wiki/ Template:COVID-19_pandemic data

[67] Micky Willmott, Alexandra Nicholson, Heide Busse, Georgina J MacArthur, Sara Brookes, and Rona Campbell. 2016. Effectiveness of hand hygiene interventions in reducing illness absence among children in educational settings: a systematic review and meta-analysis. Archives of disease in childhood 101, 1 (2016), 42-50.

[68] World Health Organization. 2019. WHO guideline: recommendations on digital interventions for health system strengthening: web supplement 2: summary of findings and GRADE tables. No. WHO/RHR/19.7 (2019).

[69] Songjie Wu, Ying Wang, Xuelan Jin, Jia Tian, Jianzhong Liu, and Yiping Mao. 2020. Environmental contamination by SARS-CoV-2 in a designated hospital for coronavirus disease 2019. American fournal of Infection Control (2020).

[70] Songjie Wu, Ying Wang, Xuelan Jin, Jia Tian, Jianzhong Liu, and Yiping Mao. 2020. Environmental contamination by SARS-CoV-2 in a designated hospital for coronavirus disease 2019. American fournal of Infection Control (2020). https: //doi.org/10.1016/j.ajic.2020.05.003 


\section{APPENDIX}

\section{A QUESTIONNAIRE: PUBLIC HEALTH KNOWLEDGE COVID-19 (PHKC)}

(1) Masks should be worn by everyone regardless of age:

(a) True

(b) False

(2) Masks should cover a person's:
(a) Mouth
(b) Nose
(c) Both (a) and (b).

(3) Which of the following are currently known symptoms of COVID-19? (Select all that apply)
(a) Dry cough
(b) Fatigue
(c) Loss of taste or smell
(d) Fever
(e) Feeling energized
(f) Seizures
(g) Runny nose

(4) Which of the following is a proper way to wash your hands?

(a) Wet your hands with clean water, apply low concentration bleach, rinse your hands for 30 seconds, then dry your hands.

(b) Apply soap to your hands, then wash your hands under running water for 20 seconds, then dry your hands.

(c) Wet your hands with clean water, apply soap and scrub your hands for 20 seconds, rinse your hands, then dry your hands.

(d) Wet your hands with clean water, apply soap, rinse your hands for 30 seconds, then dry your hands.

(5) What is the incubation period for coronavirus?
(a) 9 months
(b) 1 to 2 days
(c) 2 to 4 days
(d) 2 to 7 days
(e) 2 to 4 weeks
(f) 2 days to 2 weeks
(g) Immediately

\section{B QUESTIONNAIRE: SHARED WORKSPACE COVID-19 KNOWLEDGE (SWCK)}

(1) How long does the coronavirus survive on the surfaces of plastic keyboards or countertops?
(a) Up to two weeks
(b) Several hours
(c) Up to three days
(d) Up to nine days

(2) Which of the following is the minimum acceptable alcohol concentration for cleaning your computer workstation?
(a) $40 \%$
(b) $50 \%$
(c) $55 \%$
(d) $60 \%$
(e) $65 \%$
(f) $70 \%$

(3) For effective social distancing, you should remain at least (a) 3 feet away from other people.

(b) 4 (c) 5

(d) 6

(e) 7

(f) 8

(4) List all the frequently touched areas in this lab:

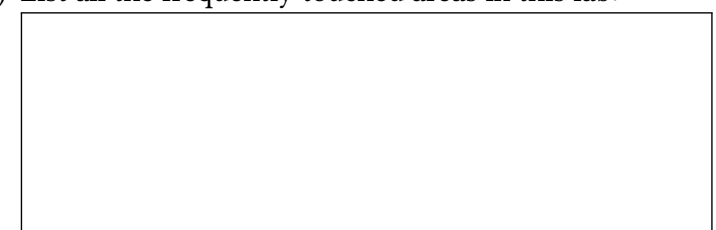

\section{QUESTIONNAIRE: COVID-19 HYGIENIC WORKSTATION SELF-EFFICACY}

(1) I am confident I can recognize appropriate cleaning solutions to use to sanitize computer workstations from COVID-19.

(a) 1 (Strongly Disagree)

(b) 2 (Disagree)

(c) 3 (Neither Disagree nor Agree)

(d) 4 (Agree)

(e) 5 (Strongly Agree)

(2) I am confident I can sanitize a computer workstation correctly.
(a) 1 (Strongly Disagree)
(b) 2 (Disagree)
(c) 3 (Neither Disagree nor Agree)
(d) 4 (Agree)
(e) 5 (Strongly Agree)

(3) I am confident that I can identify frequently touched spots on a shared computer workstation.
(a) 1 (Strongly Disagree)
(b) 2 (Disagree)
(c) 3 (Neither Disagree nor Agree)
(d) 4 (Agree)
(e) 5 (Strongly Agree)

(4) I am confident in my ability to social distance while using a shared computer workstation.
(a) 1 (Strongly Disagree)
(b) 2 (Disagree)
(c) 3 (Neither Disagree nor Agree)
(d) 4 (Agree)
(e) 5 (Strongly Agree)

(5) I am confident in my ability to wear a mask effectively while using a shared computer workstation.
(a) 1 (Strongly Disagree)
(b) 2 (Disagree)
(c) 3 (Neither Disagree nor Agree)
(d) 4 (Agree)
(e) 5 (Strongly Agree)

(6) I am confident in my ability to wash my hands effectively after using a shared computer workstation.
(a) 1 (Strongly Disagree)
(b) 2 (Disagree)
(c) 3 (Neither Disagree nor Agree)
(d) 4 (Agree)
(e) 5 (Strongly Agree) 


\section{QUESTIONNAIRE: COVID-19 ANXIETY SCALE}

(1) I am worried about catching COVID-19 through contact with shared computer workstations (such as desk, chairs, keyboard and/or mouse).
(a) 1 (Not At All)
(b) 2 (Rarely)
(c) 3 (Sometimes)
(d) 4 (Often)
(e) 5 (Always)

(2) I am worried about accidentally touching my face while my hands have been in contact with frequently touched surfaces.
(a) 1 (Not At All)
(b) 2 (Rarely)
(c) 3 (Sometimes)
(d) 4 (Often)
(e) 5 (Always)

\section{E QUESTIONNAIRE: COVID-19 POSITIVE HYGIENIC ATTITUDES}

(1) Washing hands is essential to protect myself from COVID-19.
(a) 1 (Strongly Disagree)
(b) 2 (Disagree)
(c) 3 (Neither Disagree nor Agree)
(d) 4 (Agree)
(e) 5 (Strongly Agree)

(2) COVID-19 will eventually be successfully controlled.
(a) 1 (Strongly Disagree)
(b) 2 (Disagree)
(c) 3 (Neither Disagree nor Agree)

(d) 4 (Agree)

(e) 5 (Strongly Agree)

(3) Proper cleaning and disinfecting measures in workstations can help win the battle against COVID-19.
(a) 1 (Strongly Disagree)
(b) 2 (Disagree)
(c) 3 (Neither Disagree nor Agree)
(d) 4 (Agree)
(e) 5 (Strongly Agree)

(4) Shared computer workstations (such as the ones in this lab) are usually germ-free.
(a) 1 (Strongly Disagree)
(b) 2 (Disagree)
(c) 3 (Neither Disagree nor Agree)
(d) 4 (Agree)
(e) 5 (Strongly Agree)

(5) I frequently disinfect shared computer workstations (such as the keyboard and mouse) before using them.
(a) 1 (Strongly Disagree)
(b) 2 (Disagree)
(c) 3 (Neither Disagree nor Agree)
(d) 4 (Agree)
(e) 5 (Strongly Agree)

(6) I frequently disinfect shared computer workstations (such as the keyboard and mouse) after using them.
(a) 1 (Strongly Disagree)
(b) 2 (Disagree)
(c) 3 (Neither Disagree nor Agree)
(d) 4 (Agree)
(e) 5 (Strongly Agree) 


\section{F RUBRIC: CLEANING BEHAVIOR}

\begin{tabular}{|c|c|c|c|c|c|}
\hline & Highly Unsafe Practice & Unsafe Practice & Less Unsafe Practice & Safe Practice & Ideal Practice \\
\hline Rubric & 1 & $\overline{2}$ & 3 & $\overline{4}$ & 5 \\
\hline $\begin{array}{l}\text { Cleaning before } \\
\text { use }\end{array}$ & $\begin{array}{l}\text { No cleaning. Immediately } \\
\text { started using workstation. }\end{array}$ & $\begin{array}{l}\text { General cleaning such as } \\
\text { rearranging desktop } \\
\text { set-up, using a new chair } \\
\text { which is clean, using hand } \\
\text { sanitizer only on self, } \\
\text { using new or personal } \\
\text { headphones. No visible } \\
\text { use of disinfectants. }\end{array}$ & $\begin{array}{l}\text { Cleaning with } \\
\text { disinfectants. Cleaning } \\
\text { the general open surface } \\
\text { of the desk. Cleaning did } \\
\text { not cover areas under the } \\
\text { keyboard, mouse, the } \\
\text { monitor surface, and the } \\
\text { CPU power button. }\end{array}$ & $\begin{array}{l}\text { Cleaning with } \\
\text { disinfectants. Cleaning } \\
\text { covered the general open } \\
\text { surface of the desk } \\
\text { including the surface of } \\
\text { the keyboard and the } \\
\text { mouse. Cleaning covered } \\
\text { the areas under the } \\
\text { mouse and keyboard. } \\
\text { Cleaning did not cover } \\
\text { the monitor surface and } \\
\text { CPU power button. }\end{array}$ & $\begin{array}{l}\text { Cleaning with } \\
\text { disinfectants. Cleaning } \\
\text { general open surface of } \\
\text { the desk including the } \\
\text { surface of the keyboard } \\
\text { and the mouse. Cleaning } \\
\text { covered the areas under } \\
\text { the mouse and keyboard. } \\
\text { Covered all areas } \\
\text { including the monitor } \\
\text { surface and the CPU } \\
\text { power button. Thorough } \\
\text { cleaning. }\end{array}$ \\
\hline $\begin{array}{l}\text { Face mask } \\
\text { wearing }\end{array}$ & No face covering. & $\begin{array}{l}\text { Face was occasionally } \\
\text { covered. }\end{array}$ & $\begin{array}{l}\text { Mask worn at all times } \\
\text { but exposed nose or } \\
\text { mouth frequently. }\end{array}$ & $\begin{array}{l}\text { Mask worn at all times } \\
\text { but exposed nose or } \\
\text { mouth once or twice. }\end{array}$ & $\begin{array}{c}\text { Mask worn properly at all } \\
\text { times. }\end{array}$ \\
\hline $\begin{array}{l}\text { Absence of eyes, } \\
\text { nose or mouth } \\
\text { touching }\end{array}$ & $\begin{array}{l}\text { Frequent touching of } \\
\text { eyes, nose, or mouth } \\
\text { without sanitizing hands. }\end{array}$ & $\begin{array}{l}\text { Occasionally touched } \\
\text { eyes, nose, or mouth } \\
\text { without sanitizing hands. }\end{array}$ & $\begin{array}{l}\text { Occasionally touched } \\
\text { eyes, nose, or mouth after } \\
\text { sanitizing hands. }\end{array}$ & $\begin{array}{c}\text { No touching eyes, nose, } \\
\text { or mouth. No sanitizing } \\
\text { of hands. }\end{array}$ & $\begin{array}{c}\text { No touching eyes, nose, } \\
\text { or mouth. Hands were } \\
\text { sanitized. }\end{array}$ \\
\hline $\begin{array}{c}\text { Cleaning after } \\
\text { use }\end{array}$ & No cleaning. & $\begin{array}{l}\text { Cleaned with or without } \\
\text { disinfectants. Small } \\
\text { number of cleaning } \\
\text { strokes. Swiped the desk } \\
\text { one or two times. } \\
\text { Cleaning did not cover all } \\
\text { the areas including under } \\
\text { the keyboard and the } \\
\text { mouse. Cleaned for an } \\
\text { insufficient amount of } \\
\text { time. }\end{array}$ & $\begin{array}{c}\text { Cleaned with } \\
\text { disinfectants. Cleaning } \\
\text { covered the entire } \\
\text { workspace, including } \\
\text { under the keyboard and } \\
\text { the mouse. Small number } \\
\text { of cleaning strokes. } \\
\text { Swiped the desk just one } \\
\text { or two times. Cleaned for } \\
\text { an insufficient amount of } \\
\text { time. }\end{array}$ & $\begin{array}{c}\text { Cleaned with } \\
\text { disinfectants. Cleaning } \\
\text { covered the entire area } \\
\text { including under the } \\
\text { keyboard and the mouse. } \\
\text { Wiped surfaces in an } \\
\text { orderly manner } \\
\text { numerous times. Cleaned } \\
\text { for an insufficient amount } \\
\text { of time. }\end{array}$ & $\begin{array}{c}\text { Cleaned with } \\
\text { disinfectants. Cleaning } \\
\text { covered the entire area } \\
\text { including under the } \\
\text { keyboard and the mouse. } \\
\text { Wiped surfaces in an } \\
\text { orderly manner } \\
\text { numerous times. Cleaned } \\
\text { the surfaces for an } \\
\text { adequate amount of time. }\end{array}$ \\
\hline $\begin{array}{c}\text { Total cleaning } \\
\text { time } \\
\text { (pre-cleaning } \\
\text { time }+ \\
\text { post-cleaning } \\
\text { time) }\end{array}$ & 0 seconds. & 1-5 seconds. & 5-10 seconds. & $10-15$ seconds. & $>15$ seconds. \\
\hline
\end{tabular}




\section{G TABLES}

Table 2: Player Experience Inventory scores for the in-person study.

\begin{tabular}{|c|c|c|c|c|c|c|}
\hline \multicolumn{2}{|c|}{ Subscales } & \multirow{2}{*}{$\frac{M}{2.06}$} & \multirow{2}{*}{$\frac{S D}{0.83}$} & \multirow{2}{*}{$\frac{\text { Min }}{-3.00}$} & \multirow{2}{*}{$\frac{\text { Max }}{3.00}$} & \multirow{2}{*}{$\frac{\alpha}{0.88}$} \\
\hline 1 & Audiovisual Appeal & & & & & \\
\hline 2 & Autonomy & -1.50 & 0.75 & -3.00 & 3.00 & 0.61 \\
\hline 3 & Challenge & -0.33 & 1.85 & -3.00 & 3.00 & 0.90 \\
\hline 4 & Clarity of Goals & 2.78 & 0.40 & -3.00 & 3.00 & 0.89 \\
\hline 5 & Curiosity & 1.22 & 1.46 & -3.00 & 3.00 & 0.96 \\
\hline 6 & Ease of Control & 2.00 & 1.28 & -3.00 & 3.00 & 0.91 \\
\hline 7 & Enjoyment & 1.56 & 1.38 & -3.00 & 3.00 & 0.98 \\
\hline 8 & Immersion & 0.72 & 1.51 & -3.00 & 3.00 & 0.95 \\
\hline 9 & Mastery & 1.94 & 1.10 & -3.00 & 3.00 & 0.37 \\
\hline 10 & Meaning & 1.78 & 0.96 & -3.00 & 3.00 & 0.97 \\
\hline 11 & Progress Feedback & 2.22 & 0.89 & -3.00 & 3.00 & 0.78 \\
\hline
\end{tabular}

Table 3: Video Engagement Scale scores for the in-person study.

\begin{tabular}{|c|c|c|c|c|c|}
\hline Subscales & $M$ & $\mathrm{SD}$ & Min & Max & $\alpha$ \\
\hline 1 Attention & 4.47 & 0.87 & 1.00 & 7.00 & 0.33 \\
\hline 2 Emotions & 2.87 & 1.07 & 1.00 & 7.00 & 0.76 \\
\hline 3 Going Into a Narrative World & 2.67 & 1.13 & 1.00 & 7.00 & 0.68 \\
\hline
\end{tabular}

Table 4: Participant distribution by race/ethnicity in online study.

\begin{tabular}{rlr}
\hline Race & $\mathrm{N}$ \\
\hline 1 & White & 241 \\
2 & Black or African American & 34 \\
3 & Asian Indian & 7 \\
4 & Chinese & 7 \\
5 & Other & 6 \\
6 & Filipino & 3 \\
7 & Other Asian & 3 \\
8 & American Indian or Alaska Native & 1 \\
9 & Japanese & 1 \\
10 & Native Hawaiian & 1 \\
11 & Vietnamese & 1 \\
\hline
\end{tabular}

Table 5: Participant distribution by highest education attained in online study.

\begin{tabular}{llr}
\hline Education & $\mathrm{N}$ \\
\hline 1 & High School & 31 \\
2 & Some college, no degree & 28 \\
3 & Associate's Degree & 28 \\
4 & Bachelor's Degree & 158 \\
5 & Graduate Degree (Master's, Doctoral) & 56 \\
6 & Professional Degree (law, medical, dental) & 4 \\
\hline
\end{tabular}


Table 6: Participant distribution by household income in online study.

\begin{tabular}{lcr}
\hline Household Income & $\mathrm{N}$ \\
\hline 1 & Less than $\$ 12,500$ & 9 \\
2 & $\$ 12,500-\$ 24,999$ & 38 \\
3 & $\$ 25,000-\$ 37,499$ & 34 \\
4 & $\$ 37,500-\$ 49,999$ & 60 \\
5 & $\$ 50,000-\$ 62,499$ & 49 \\
6 & $\$ 62,500-\$ 74,999$ & 32 \\
7 & $\$ 75,000-\$ 87,499$ & 25 \\
8 & $\$ 87,500-\$ 99,999$ & 26 \\
9 & $\$ 100,000$ or more & 32 \\
\hline
\end{tabular}

Table 7: Player Experience Inventory scores for the online study.

\begin{tabular}{|c|c|c|c|c|c|c|}
\hline \multicolumn{2}{|c|}{ Subscales } & \multirow{2}{*}{$\frac{\mathrm{M}}{1.81}$} & \multirow{2}{*}{$\begin{array}{c}\mathrm{SD} \\
1.22\end{array}$} & \multirow{2}{*}{$\frac{\text { Min }}{-3.00}$} & \multirow{2}{*}{$\frac{\text { Max }}{3.00}$} & \multirow{2}{*}{$\frac{\alpha}{0.92}$} \\
\hline 1 & Audiovisual Appeal & & & & & \\
\hline 2 & Autonomy & 0.49 & 1.80 & -3.00 & 3.00 & 0.93 \\
\hline 3 & Challenge & 0.84 & 1.45 & -3.00 & 3.00 & 0.83 \\
\hline 4 & Clarity of Goals & 2.19 & 0.89 & -3.00 & 3.00 & 0.86 \\
\hline 5 & Curiosity & 1.51 & 1.43 & -3.00 & 3.00 & 0.92 \\
\hline 6 & Ease of Control & 1.96 & 1.04 & -3.00 & 3.00 & 0.85 \\
\hline 7 & Enjoyment & 1.73 & 1.31 & -3.00 & 3.00 & 0.93 \\
\hline 8 & Immersion & 1.34 & 1.25 & -3.00 & 3.00 & 0.72 \\
\hline 9 & Mastery & 1.82 & 1.15 & -3.00 & 3.00 & 0.85 \\
\hline 10 & Meaning & 1.71 & 1.32 & -3.00 & 3.00 & 0.92 \\
\hline & Progress Feedback & 1.88 & 1.14 & -3.00 & 3.00 & 0.85 \\
\hline
\end{tabular}

Table 8: Video Engagement Scale scores for the online study.

\begin{tabular}{|c|c|c|c|c|c|}
\hline Subscales & $M$ & $\mathrm{SD}$ & Min & $\operatorname{Max}$ & $\alpha$ \\
\hline 1 Attention & 5.59 & 1.05 & 1.00 & 7.00 & 0.56 \\
\hline 2 Emotion & 4.50 & 1.60 & 1.00 & 7.00 & 0.83 \\
\hline 3 Going Into a Narrative World & 4.94 & 1.46 & 1.00 & 7.00 & 0.78 \\
\hline
\end{tabular}


Table 9: Missingness regression results showing $\beta$ and standard error in parentheses. To avoid overparameterization, the first level of categorical variables is automatically deleted when fitting the regression equation [25]. As a result, "White" (race), "female" (gender), “Less than \$12,500" (household income), and "Some High School” (education) are not present in the regression output, and the other levels of each variable are interpreted in relation to the first level of the respective categorical variable.

\begin{tabular}{|c|c|c|}
\hline \multirow[b]{3}{*}{ Age } & \multirow{2}{*}{\multicolumn{2}{|c|}{$\begin{array}{c}\text { Dependent variable: } \\
\text { Missing Value }\end{array}$}} \\
\hline & & \\
\hline & $-0.040^{*}$ & $(0.016)$ \\
\hline Gender: Male & -0.109 & $(0.314)$ \\
\hline Gender: Other & -16.127 & $(2,659.586)$ \\
\hline Household Income: $\$ 12,500$ - $\$ 24,999$ & -1.317 & $(1.110)$ \\
\hline Household Income: $\$ 25,000-\$ 37,499$ & 0.344 & $(0.905)$ \\
\hline Household Income: $\$ 37,500$ - $\$ 49,999$ & -0.333 & $(0.911)$ \\
\hline Household Income: $\$ 50,000-\$ 62,499$ & -0.289 & $(0.921)$ \\
\hline Household Income: $\$ 62,500-\$ 74,999$ & -0.263 & $(0.945)$ \\
\hline Household Income: $\$ 75,000-\$ 87,499$ & 0.142 & $(0.937)$ \\
\hline Household Income: $\$ 87,500$ - $\$ 99,999$ & -0.747 & $(1.054)$ \\
\hline Household Income: $\$ 100,000$ or more & -2.351 & $(1.333)$ \\
\hline Race: Black or African American & -17.376 & $(2,797.610)$ \\
\hline Race: Asian Indian & 10.899 & $(2,611.990)$ \\
\hline Race: Chinese & -0.493 & $(2,776.419)$ \\
\hline Race: Other & -5.387 & $(2,651.260)$ \\
\hline Race: Filipino & 5.173 & $(2,684.263)$ \\
\hline Race: Other Asian & -0.014 & $(2,749.818)$ \\
\hline Race: American Indian or Alaska Native & -2.341 & $(2,553.676)$ \\
\hline Race: Japanese & 4.912 & $(2,759.357)$ \\
\hline Race: Native Hawaiian & -3.321 & $(2,615.766)$ \\
\hline Race: Vietnamese & 1.554 & $(2,257.202)$ \\
\hline Education: Some college, no degree & -0.153 & $(0.838)$ \\
\hline Education: Associate’s degree & 0.638 & $(0.767)$ \\
\hline Education: Bachelor's degree & 0.483 & $(0.612)$ \\
\hline Education: Graduate degree (Master's, Doctorate) & 0.936 & $(0.672)$ \\
\hline Education: Professional degree (Law, Medical, Dental) & 1.975 & $(1.126)$ \\
\hline Experimental Condition & -0.188 & $(0.314)$ \\
\hline Constant & -11.439 & $(799.012)$ \\
\hline Observations & & 62 \\
\hline Log Likelihood & -13 & 9.612 \\
\hline Akaike Inf. Crit. & & .224 \\
\hline
\end{tabular}

Note: ${ }^{*} \mathrm{p}<0.05 ;{ }^{* *} \mathrm{p}<0.01 ;{ }^{* * *} \mathrm{p}<0.001$ 